\title{
3. Komplementärmedizin in der Onkologie
}

\author{
HANS-WOLFGANG HOEFERT UND BERNHARD UEHLEKE
}

\section{Einleitung}

Krebserkrankungen stehen in der Bevölkerung in dem Ruf, plötzlich und ohne erkennbare Ursache aufzutreten und im weiteren Verlauf, an dessen Ende ein qualvoller Tod befürchtet wird, kaum kontrollierbar zu sein. Für Viele scheint Krebs die Haupttodesursache hierzulande zu sein. Tatsächlich aber sterben mehr als die Hälfte aller Menschen an Herz-Kreislauf-Erkrankungen und nur jeder Fünfte stirbt an Krebs. Dennoch veranlasst der besondere Charakter von Krebserkrankungen viele Menschen, bereits präventiv zu Mitteln der Komplementärmedizin (KM) zu greifen und mehr als die Hälfte bedient sich der KM, wenn eine Krebserkrankung eingetreten ist. Im Folgenden soll zunächst beschrieben werden, welche Menschen besonders stark von KM Gebrauch machen und welche Mittel und Methoden gewählt werden. Die Motive für den Gebrauch reichen dabei von einer direkten Krebsbekämpfung über die Stärkung des Immunsystems, Erhaltung eines positiven Lebensgefühls bis hin zur Abschwächung von Symptomen, wie sie als Begleiterscheinung der Erkrankung selbst oder ihrer konventionellen Therapie auftreten. Wir beschränken uns in diesem Kapitel angesichts der Vielfalt von Krebstypen auf den bei Frauen am häufigsten vertretenen Brustkrebs sowie auf den bei Männern am häufigsten vertretenen Prostatakrebs. 


\subsection{Epidemiologie von Krebserkrankungen und Inanspruchnahme von KM}

Die Gesundheitsstatistiken zu Krebserkrankungen in Deutschland zeigen zwei gegenläufige Trends: Einerseits steigt die Zahl der Krebserkrankungen in der älter werdenden Bevölkerung; so sind die Erkrankungsraten im Zeitraum 1980 bis 2004 bei Männern um 30\% und bei Frauen um 20\% gestiegen. Bei Frauen rangieren auf den ersten beiden Plätzen Brustkrebs und Darmkrebs, mit Abstand gefolgt von Lungenkrebs und Gebärmutterkörperkrebs. Bei Männern liegen auf den ersten drei Plätzen Prostatakrebs, Darmkrebs und Lungenkrebs, mit Abstand gefolgt von Harnblasenkrebs, Magenkrebs und Nierenkrebs (RKI 2010).

Andererseits ist die Sterblichkeit bei Männern und Frauen um etwa 20\% gesunken, sodass heute im Durchschnitt von größeren Überlebenschancen ausgegangen werden kann als noch vor 20 oder 30 Jahren $^{12}$ (RKI 2010). Man vermutet, dass diese Verbesserungen insgesamt auf einen gesünderen Lebensstil und teilweise auch weniger schädliche Umweltbedingungen (gesundheitsbewusstere Ernährung, mehr Bewegung, weniger Rauchen und weniger Giftstoff-Exposition in der Arbeitsumwelt) zurückzuführen sind.

$\mathrm{Zu}$ den Nutzern der KM bei Krebs zählen überwiegend Frauen und Personen mit einem höheren Schulabschluss, wobei die Nutzungshäufigkeit ein Jahr bis drei Jahre nach der Erstdiagnose am höchsten ist. Eine diesbezügliche Untersuchung in den USA (Garland et al. 2013) zeigt auch, dass der größte Gewinn („benefit finding“) beim sog. Energieheilen, weniger häufig bei Spezialdiäten, Akupunktur, Chiropraktik, Kräutern, Vitaminen und Massagepraktiken und kaum bei Homöopathie, Entspannungstechniken, Yoga und Tai Chi gefunden wird.

Wieweit die KM-Nutzung von den jeweiligen Krankenhäusern unterstützt wird, hängt sehr von den Einstellungen der dort Beschäftigten ab. Nach einer kleineren deutschen Studie (Trimborn et al. 2013) scheinen weibliche Beschäftigte eher daran interessiert zu sein als männliche und Pflegekräfte mehr als Ärzte. Jene haben auch größere Bedenken wegen möglicher Nebenwirkungen von KM, wobei rund $70 \%$ der Beschäftigten zugab, nicht genügend über KM informiert zu sein.

Insbesondere dann, wenn eine Krebsdiagnose gestellt wurde, aber auch im weiteren Verlauf einer chemotherapeutischen und radiologischen Behandlung sowie später, wenn die Erkrankung zum Stillstand gekommen ist, wird von Mitteln und Methoden der KM Gebrauch gemacht. Nach einer neueren Metaanalyse zu den Nutzungshäufigkeiten von KM bei Krebs, die in einschlägigen Untersuchungen aus 18 Ländern bis 2009 berichtet wurden, beträgt die durchschnittliche Nutzungsrate während oder nach einer Krebsbehandlung etwa 40\%. Die höchsten Häufigkeiten wurden in den USA, die niedrigsten in Italien und den Niederlan-

\footnotetext{
${ }^{12}$ Die Überlebensraten für Magen- und Lungenkrebs bei Männern und für Krebs an den Geschlechtsorganen bei Frauen haben sich deutlich gebessert, während die Sterberaten für Speiseröhren- und Bauchspeicheldrüsenkrebs sowie für Lungenkrebs bei Frauen gestiegen sind (RKI 2010).
} 
den gefunden. Insgesamt ist die Nutzungsrate von $25 \%$ in den $70 e r-J a h r e n$ auf 49\% nach dem Jahr 2000 angestiegen (vgl. Horneber et al. 2012). Ein direkter Vergleich der einzelnen Untersuchungsergebnisse wird aus methodologischen Gründen erschwert ${ }^{13}$, was sich bereits im innereuropäischen Vergleich oder bei Studien ein und desselben Landes zeigt. So variieren die Nutzungsraten innerhalb Europas beispielsweise zwischen rund 15 und 73\% mit einem Durchschnitt von $36 \%$ (Molassiotis et al. 2005). Die relativ große Varianz der Nutzungsraten in den erwähnten Studien dürfte auch darauf zurückzuführen sein, dass nicht zwischen den jeweils repräsentierten Krebsarten differenziert wurde. Deshalb sollen im Folgenden noch einmal die Studienergebnisse für einzelne Krebsarten näher betrachtet werden.

\subsubsection{Brustkrebs}

Brustkrebs ist die häufigste Krebsart bei Frauen, wobei mit einem weiteren Anstieg bis 2015 gerechnet werden muss. Aus unterschiedlichen möglichen Gründen (steigende Zahl von evidenzbasierten Studien zur KM, Medienberichte, Furcht Nebenwirkungen konventioneller Interventionen usw.) ist ebenfalls ein Trend zur Verwendung von KM festzustellen: So geben Boon et al. (2007) eine KM-Nutzung von 66,7\% im Jahr 1998 und von 81,9\% im Jahr 2005 an. Die erhöhte Nutzung bezieht sich sowohl auf KM-Produkte als auch auf Besuche bei einem KM-Spezialisten. Die am häufigsten verwendeten Mittel oder Methoden sind in den USA dieser Analyse zufolge grüner Tee, Vitamin E, Flachssamen, Vitamin C, Massage und Nahrungsmittelzusätze (s. Tab. 12).

Tab. 12 Studien zur Verwendung von KM bei aktuellem Brustkrebs aus den letzten 10 Jahren

\begin{tabular}{ll} 
Studie & Ergebnisse in Kurzform \\
\hline $\begin{array}{l}\text { Tautz et al. } 2012 \\
\text { Deutschland }\end{array}$ & $\begin{array}{l}\text { Prädiktoren sind jüngeres Alter und höherer Bildungsgrad } \\
\text { Nutzung von KM durch Hinweise aus der Familie von Freunden, vom Allgemeinarzt oder } \\
\text { aus den Medien } \\
\text { Gründe für den Nicht-Gebrauch von KM: konventionelle Medizin ausreichend (34\%), noch } \\
\text { nicht über KM nachgedacht (31\%) }\end{array}$ \\
\end{tabular}

\footnotetext{
${ }^{13}$ Horneber et al. (2012) verweisen hier beispielsweise auf die unterschiedlichen Befragungsmethoden (Fragebogen, Direktinterview), welche zu Unter- bzw. Überschätzungen führen können, auf die mehr oder weniger weiten KM-Definitionen, auf die unterschiedlichen Untersuchungssettings (Telefonbefragung, Befragung während eines Klinikaufenthaltes u.ä.) sowie auf die unterschiedlichen Zeiträume, auf die sich der KM-Gebrauch bezieht.
} 


\begin{tabular}{|c|c|}
\hline Studie & Ergebnisse in Kurzform \\
\hline Saquib et al. 2011 & $80 \%$ nutzen KM aus allgemeinen Gründen, nur 50\% aus krebsspezifischen Gründen. \\
\hline USA & $\begin{array}{l}\text { Nutzerinnen sind eher jünger, haben einen höheren Bildungsgrad, einen höheren Ver- } \\
\text { brauch an Obst und Gemüse und einen niedrigeren Body-Mass-Index (BMI) } \\
\text { KM-Nutzerinnen berichten über stärkere psychische und körperliche Beeinträchtigungen } \\
\text { als Nicht-Nutzerinnen. }\end{array}$ \\
\hline Wanchai et al. 2010 & Brustkrebs-Patientinnen nutzen KM mehr als Pat. mit anderen Krebsarten. \\
\hline USA & $\begin{array}{l}\text { An vorderster Stelle werden biologische Verfahren genutzt, danach Mind-Body-Verfahren, } \\
\text { philosophisch umfassende Verfahren und Energietechniken. } \\
\text { KM-Nutzerinnen sind eher jünger, haben eine höhere Bildung und ein höheres Einkom- } \\
\text { men, sind eher verheiratet und Mitglied einer Selbsthilfegruppe. } \\
\text { Nur 50\% sprechen mit ihrem Arzt über den KM-Gebrauch. }\end{array}$ \\
\hline Greenlee et al. 2009 & $86 \%$ der Patientinnen nutzen KM im Anschluss an die Erstdiagnose. \\
\hline USA & $\begin{array}{l}\text { Die häufigsten Behandlungsformen sind grüner Tee, Glokusamine, Omega-3-Fettsäuren } \\
\text { und Beten. } \\
\text { KM-Gebrauch vor und nach der Diagnose ist assoziiert mit anderen gesunden Verhal- } \\
\text { tensweisen (Obst- und Gemüseverbrauch, geringerer BMI). }\end{array}$ \\
\hline Pedersen et al. 2009 & 40,1\% der Frauen nutzen KM 12-16 Wochen nach der Erstdiagnose. \\
\hline Dänemark & $\begin{array}{l}\text { Sie sind jünger als Nicht-Nutzerinnen, haben ein höheres Bildungs- und Einkommensni- } \\
\text { veau, eine geringe Komorbidität, leben eher allein oder getrennt, haben eher ein Nor- } \\
\text { malgewicht, sind überwiegend Nicht-Raucher und leben eher in größeren Städten. } \\
\text { Ca. } 34 \% \text { glauben, dass KM-Nutzung einen positiven Einfluss auf ihren Brustkrebs hat. }\end{array}$ \\
\hline Matthews et al. & $69 \%$ nutzen KM noch ein Jahr nach der Erstdiagnose. \\
\hline 2007 & Sie sind eher jünger und ihre Erstdiagnose erfolgte in jüngeren Jahren. \\
\hline USA & $\begin{array}{l}\text { Ihre habituelle Angst (trait anxiety) liegt höher, insbesondere dann, wenn sie mit dem } \\
\text { Krebs in Verbindung gebracht wird. }\end{array}$ \\
\hline Molassiotis et al. & $44,7 \%$ nutzen KM seit ihrer Erstdiagnose. \\
\hline $\begin{array}{l}2006 \\
11 \text { europ. Länder }\end{array}$ & $\begin{array}{l}\text { Die am häufigsten genutzten KM-Verfahren sind Kräutermedizin und medizinische Tees, } \\
\text { danach Entspannungs- und spirituelle Verfahren, Homöopathie sowie Vitamine und } \\
\text { Mineralien. } \\
\text { KM-Nutzerinnen sind eher jünger und haben einen höheren Bildungsgrad. } \\
\text { Die meisten Nutzerinnen sind mit KM sehr zufrieden (6,5\% nicht). } \\
\text { Informationsquellen über KM sind hauptsächlich Freunde und Verwandte sowie Medien. }\end{array}$ \\
\hline
\end{tabular}




\begin{tabular}{|c|c|}
\hline Studie & Ergebnisse in Kurzform \\
\hline Helyer et al. 2006 & $47 \%$ nutzen $\mathrm{KM}, 12 \%$ lehnen eine konventionelle Behandlung ab. \\
\hline Canada & $\begin{array}{l}\text { Im Vergleich zu Frauen, die KM nicht nutzen, sind die Nutzerinnen eher jünger, leben in } \\
\text { festen Beziehungen und sind häufiger in einer Selbsthilfegruppe engagiert. } \\
\text { Überwiegend werden botanische Produkte, Vitamine und Nahrungsmittelzusätze ver- } \\
\text { wendet. } \\
40 \% \text { der Nutzerinnen haben auch vor der Diagnose schon von KM Gebrauch gemacht. } \\
\text { Hauptinformationsquellen über KM sind der Arzt, danach der Apotheker und Freunde. } \\
\text { KM-Nutzerinnen haben geringere Angstwerte als Nicht-Nutzerinnen. }\end{array}$ \\
\hline $\begin{array}{l}\text { Rakovitch et al. } \\
2005\end{array}$ & $\begin{array}{l}43 \% \text { nutzen KM. Sie sind eher jünger, haben einen höheren Bildungsgrad und häufiger } \\
\text { eine Vollzeitbeschäftigung als Nicht-Nutzerinnen. }\end{array}$ \\
\hline Canada & $\begin{array}{l}\text { Bezüglich der Angst- und Depressionswerte lassen sich keine Unterschiede zwischen } \\
\text { Nutzerinnen und Nicht-Nutzerinnen feststellen. }\end{array}$ \\
\hline Nagel et al. 2004 & Noch drei Jahre nach der Erstdiagnose beträgt der Nutzungsgrad von KM 36\%. \\
\hline Deutschland & $\begin{array}{l}\text { Nutzerinnen sind eher jünger und häufiger in Selbsthilfegruppen oder Freizeitaktivitäten } \\
\text { engagiert. } \\
\text { Die meisten Nutzerinnen sind durch ihren Arzt über KM informiert. }\end{array}$ \\
\hline Navo et al. 2004 & $8 \%$ nutzen KM, davon überwiegend pflanzliche Produkte, Vitamine und Mineralien. \\
\hline USA & $\begin{array}{l}\text { Die Nutzerinnen haben überwiegend einen höheren Bildungsstatus. } \\
\text { Nur ca. 54\% sprechen mit ihrem Arzt über die KM-Nutzung. }\end{array}$ \\
\hline
\end{tabular}

Es gibt nur relativ wenige Informationen darüber, welche Rolle die KM-Nutzung in der Langzeitperspektive spielt. Generell scheint KM-Nutzung nicht mit einer Brustkrebs-spezifischen Mortalität assoziiert zu sein, obwohl hier Zusammenhänge zwischen der ethnischen Zugehörigkeit ebenso wie dem Gebrauch bestimmter Methoden wie Energieheilen berichtet werden (z.B. Matsuno et al. 2012). Ein erhöhtes Mortalitätsrisiko wurde schließlich auch für überwiegende Nutzerinnen von pflanzlichen Produkten (Eccinacea, Kräutertees und Gingko) festgestellt (Ma et al. 2011). Zumindest weisen langfristig überdurchschnittlich häufige KM-Nutzerinnen höhere medizinisch relevante Komorbiditäten und ein geringeres emotionales Wohlgefühl nach dem SF-36 auf, während sie sich bezüglich des körperlichen Wohlgefühls nicht von Nicht-Nutzerinnen unterscheiden (Carpenter et al. 2009). Außerdem scheinen Überlebende - ungeachtet ihrer KMNutzung - ein deutlich verringertes Rezidiv-Risiko zu haben, wenn sie in sozial befriedigenden Beziehungen leben (Epplein et al. 2011). Da bisher keine spezifischen Risikofaktoren in der Ernährung oder im generell im Lebensstil entdeckt wurden, die das spezifische Rezidiv- oder Mortalitätsrisiko erhöhen (vgl. Kushi 2007), erscheint es nachvollziehbar, wenn ein großer Teil der Frauen in den o.e. Studien als Motiv für den KM-Gebrauch Themen wie allgemeine Lebensqualität, emotionales Wohlbefinden u.ä. nennen und auf KM nicht unbedingt mit dem 
Ziel einer direkten Krebsbekämpfung zurückgreifen. Vielmehr scheinen insbesondere KM-Methoden, welche die positiven Aspekte aus einer Krebserfahrung betonen, geeignet $\mathrm{zu}$ sein, auch das weitere Überleben positiver $\mathrm{zu}$ sehen und $\mathrm{zu}$ gestalten als in der Zeit vor der Krebsdiagnose (Casellas-Grau et al. 2013).

\subsubsection{Prostatakrebs}

Die bisher umfassendste Auswertung von Studien zur Nutzung von KM bei Prostatakrebs (Bishop et al. 2010) bezieht sich auf die Jahre 1999 bis 2009. Diesem Review zufolge variieren die Prävalenzen für KM-Verwendung zwischen 8 und 90\% (Median 30\%). Durchgängig wird der Befund bestätigt, dass KM-Nutzer ein höheres Einkommen und einen höheren Bildungsabschluss sowie einen höheren Schweregrad des Prostatakrebses haben als Nicht-Nutzer. Ebenfalls kennzeichnend für die meisten Studien, auf die sich Bishop et al. (2010) beziehen bzw. für die in der Tabelle 13 genannten Studien ist die Tatsache, dass nur etwa die Hälfte aller KM-Nutzer darüber mit dem behandelnden Arzt spricht. Die wesentlichen Gründe dafür dürften darin liegen, dass sie nicht danach gefragt werden, dass sie ihren Arzt für nicht genügend erfahren im Umgang mit KM halten oder dass sie eine schlechtere (konventionelle) Behandlung befürchten, wenn sie darüber sprechen.

Tab. 13 Studien zur Verwendung von KM bei aktuellem Prostatakrebs aus den letzten 10 Jahren

\begin{tabular}{ll}
\hline \multicolumn{1}{l}{ Studie } & Ergebnisse in Kurzform \\
\hline Ramsey et al. 2012 & $\begin{array}{l}50 \% \text { der Männer mit kürzlich diagnostiziertem Prostata-Krebs nutzen KM (39\%, } \\
\text { Wenn Beten nicht zur KM gezählt wird). } \\
\text { Zufriedenheit mit der Behandlung hängt mehr mit der Arzt-Patient- } \\
\text { Kommunikation als mit dem KM-Gebrauch zusammen. }\end{array}$ \\
\hline McDermott et al. 2012 & $\begin{array}{l}\text { 52\% nutzen ein halbes lahr nach der Diagnose KM, die meisten davon nur eine } \\
\text { USA }\end{array}$ \\
\hline Butler et al. 2011 & $\begin{array}{l}\text { Nur 43\% sprechen über den KM-Gebrauch mit ihrem behandelnden Arzt. } \\
\text { Zum Zeitpunkt der Diagnose nutzten bereits 26\% KM (in erster Linie Nahrungs- } \\
\text { mittelzusätze), sechs Monate später 11\%, wobei Nahrungsmittelzusätze weniger } \\
\text { als früher genutzt werden. } \\
\text { KM-Nutzung war häufiger bei denen, die sich entweder für Chirurgie oder für } \\
\text { Abwarten entschieden hatten. } \\
\text { Sie sind - auch im ländlichen Raum - eher jünger und haben eine gehobene } \\
\text { Bildung. }\end{array}$ \\
\hline
\end{tabular}




\begin{tabular}{|c|c|}
\hline Studie & Ergebnisse in Kurzform \\
\hline $\begin{array}{l}\text { Porter et al. } 2008 \\
\text { USA }\end{array}$ & $\begin{array}{l}\text { Der KM-Gebrauch ging um ein Viertel in den ersten zwei Jahren nach der Erstbe- } \\
\text { handlung zurück. } \\
\text { Gründe für den Verzicht waren angebliche Wirkungslosigkeit, schwierige Hand- } \\
\text { habbarkeit und Nebenwirkungen. } \\
\text { Stärker wirkende KM-Therapien wurden schneller aufgegeben als weniger wir- } \\
\text { kende. }\end{array}$ \\
\hline $\begin{array}{l}\text { Chan et al. } 2005 \\
\text { USA }\end{array}$ & $\begin{array}{l}\text { Ein Drittel der Patienten nutzte irgendeine Form von KM, davon Vitamine und } \\
\text { Mineralstoffe ( } 26 \%) \text {, Kräuter (16\%), Antioxidantien (13\%) und Prostata-spezifische } \\
\text { Mittel wie Vitamin E, Selen oder Sägepalme (12\%). } \\
\text { KM-Nutzer zeigten eine höhere Komorbidität, einen höheren diagnostischen } \\
\text { Schweregrad und hatten ein höheres Einkommen und eine höhere Bildung als } \\
\text { Nicht-Nutzer. }\end{array}$ \\
\hline $\begin{array}{l}\text { Boon et al. } 2003 \\
\text { Canada }\end{array}$ & $\begin{array}{l}\text { Rund ein Drittel aller Männer nutzen KM unmittelbar nach der Diagnose, an } \\
\text { erster Stelle natürliche Produkte wie Vitamin E, Selen und Sägepalme. } \\
\text { KM-Nutzer unterschieden sich von Nicht-Nutzern darin, dass sie häufiger Mitglied } \\
\text { in einer Selbsthilfegruppe waren, einen höheren Schweregrad aufwiesen und } \\
\text { weniger Bedenken wegen möglicher Nebenwirkungen von KM hatten. }\end{array}$ \\
\hline $\begin{array}{l}\text { Eng et al. } 2003 \\
\text { Canada }\end{array}$ & $\begin{array}{l}39 \% \text { nutzten KM, darunter vor allem Naturprodukte wie Sägepalme, Vitamin E } \\
\text { und Selen. } \\
\text { Hauptmotiv war die Stärkung des Immunsystems und die Verhinderung eines } \\
\text { Rezidivs. } \\
\text { Informationen über KM erhielten die Männer überwiegend aus dem Freundes- } \\
\text { kreis oder der Familie sowie aus dem Internet und weniger direkt von einem } \\
\text { Onkologen. } \\
\text { KM-Nutzer neigten etwas mehr dazu, eine konventionelle Behandlung hinauszu- } \\
\text { zögern oder abzubrechen als Nicht-Nutzer. }\end{array}$ \\
\hline $\begin{array}{l}\text { Ponholzer et al. } 2003 \\
\text { Österreich }\end{array}$ & $\begin{array}{l}\text { Rund } 30 \% \text { nutzten irgendeine Form von KM, zumeist eine fettreduzierte Diät, } \\
\text { Selen und Vitamin E. } \\
\text { KM-Nutzer schätzen ihre Lebensqualität geringer ein als Nicht-Nutzer. }\end{array}$ \\
\hline
\end{tabular}

Wie auch bei anderen Krebsarten wird das gesamte Spektrum von KM-Methoden auch bei Prostata-Krebs empfohlen ${ }^{14}$ bzw. in Anspruch genommen. Die Hoffnungen, die sich mit dem KM-Gebrauch verbinden und die auch von vielen konventionellen Medizinern genährt werden, sind im Wesentlichen die Verbesserung der Lebensqualität und die Stärkung der Immunfunktion durch die Nutzung von KM-Methoden oder -Mitteln. Ob diese Hoffnungen objektiv berechtigt sind, steht - zumindest im Hinblick auf Phytopharmaka - dahin (vgl. Perabo et al.

\footnotetext{
${ }^{14}$ Im kurzen Überblick: www.prostata.de
} 
2009); den entsprechenden Forschungsbedarf haben kürzlich Klempner \& Bubley (2012) charakterisiert.

\subsection{3. Überleben von Krebs und KM-Nutzung}

Objektiv steigen die Überlebensraten bei den hier diskutierten und anderen Krebsarten (mehr als 5 Jahre Überlebenszeit) bei weiterhin steigenden absoluten Zahlen bzw. jährlichen Inzidenzen; so liegt die Überlebenswahrscheinlichkeit in Deutschland bei weiblichen Krebspatienten bei 62\%, bei männlichen Patienten bei 57\% (vgl. Schilling \& Arnold 2012). Festzustehen scheint, dass nach der Erstdiagnose zunächst die KM-Nutzung ansteigt, allerdings bei Krebs nicht anders als bei anderen chronischen Erkrankungen (Mao et al. 2007). Auch scheint die spirituelle Dimension des eigenen Lebens generell an Bedeutung zuzunehmen, wobei ein Zuwachs an spirituellen Bedürfnissen auch mit stärkerer KM-Nutzung einhergeht (Mao et al. 2010), über die dann auch häufiger als zuvor mit dem jeweils behandelnden Arzt gesprochen wird (Mao et al. 2011). Ob die allgemeine Lebensqualität durch KM-Nutzung ansteigt, ist eine nicht eindeutig zu beantwortende Frage, da sich diesbezüglich KM-Nutzer und Nicht-Nutzer im Vergleich der Studien nicht klar genug unterscheiden (Shneerson et al. 2013). Abgesehen davon, dass die jeweils verwendeten Maße für „Lebensqualität“ sich häufig voneinander unterscheiden, sollte auch in Betracht gezogen werden, dass KM-Nutzung manchmal lediglich „technisch“ und manchmal auch „philosophisch-spirituell“ erfolgt und damit unterschiedliche Einstellungen zum Leben bzw. Überleben resultieren. Darüber hinaus können KM-Methoden keine Psychotherapie ersetzen, die erforderlich wäre bei Ängsten und Depressionen, die schon vor der Krebserkrankung bestanden und in der Überlebensphase weiter bestehen (siehe unten).

Unter den Krebs-Überlebenden wird KM stärker von Frauen als von Männern genutzt, wobei Frauen eher „weichere“ Methoden (Berührung, Körperempfindung u.ä.) bevorzugen, Männer dagegen eher „biologische“ Methoden, und zwar ungeachtet der jeweiligen Effektivität. Anders als man eigentlich aufgrund der wachsenden Morbidität mit dem Alter erwarten sollte, nimmt der KM-Gebrauch jedoch mit zunehmendem Alter ab (vgl. Gansler et al. 2008). Aus der o.e. Studie von Porter et al. (2008) lässt sich entnehmen, dass Männer mit Prostatakrebs die KM-Nutzung recht schnell einstellen, wenn sich keine eindeutigen Effekte zeigen. Allerdings scheint sich dann, wenn ein Überlebender die Überzeugung hat, den Krebs - mit oder ohne KM - aktiv bekämpft oder „besiegt“ zu haben, das allgemeine Lebensgefühl zu verbessern (Belizzi \& Blank 2007). 


\subsection{Merkmale und Motive von KM-Nutzern bei Krebs}

Nutzerinnen und Nutzer von KM bei Krebs unterscheiden sich in den wesentlichen Merkmalen (eher weiblich, höherer Bildungsstatus, eher älter) nicht von denjenigen der Allgemeinbevölkerung bzw. denjenigen, welche an anderen Erkrankungen leiden (vgl. Schernhammer et al. 2009). Auch scheinen Krebserkrankte nicht häufiger einen KM-Spezialisten aufzusuchen als Personen, die nicht an Krebs erkrankt sind, oder die eine Herz-Kreislauf-Erkrankung haben (Kristoffersen et al. 2012). Generell scheinen die typischen KM-Nutzer eher weiblich, vergleichsweise wohlhabend und unter 50 Jahre alt $\mathrm{zu}$ sein (vgl. Damery et al. 2011). Auch Kinder und Jugendliche, die von KM Gebrauch machen, haben Eltern mit einem höheren elterlichen Bildungsstatus und leben in einem Haushalt mit einem überdurschschnittlichen Familieneinkommen (Gottschling et al. 2013).

Eine andere Frage ist, ob Nutzer/innen von KM bei Krebs psychisch vulnerabler sind. Ein solcher Zusammenhang wurde bereits in der Studie von Paltiel et al. (2001) behauptet, in der ein Zusammenhang zwischen allgemeiner Stressbelastung (begleitende Psychotherapie, Hilflosigkeit, schlechter emotionaler und sozialer Funktionsstatus) und KM-Nutzung erkannt worden war. Ähnlich fanden Montazeri et al. (2005) eine häufigere KM-Nutzung bei Brustkrebspatientinnen mit Depressionen. Rakovitch et al. (2005) konnten dagegen keinen Zusammenhang zwischen Angst oder Depression und KM-Nutzung ermitteln; bei KMNutzerinnen sei allerdings eine stärkere Rezidiv- und Todesangst ausgeprägt. Eine taiwanesische Studie (Ku \& Koo 2012) stellt dagegen fest, dass keine psychischen Merkmale, sondern lediglich Ermüdungssymptome kennzeichnend für die Nutzergruppe seien. Ähnlich kennzeichnen Yun et al. (2013) KM-Nutzer/innen als Menschen mit schlechterer kognitiver Funktionalität und stärkerer Ermüdungsneigung. KM-Nutzer/innen scheinen andererseits bei Krebs über mehr „Kampfgeist“ und eine stärkere internale Attributionsneigung zu verfügen (Davidson et al. 2004), hohe Erwartungen an die Wirkung von KM zu stellen und sich auch stärker an den Vorschlägen aus ihrer sozialen Umgebung zu orientieren als Nicht-Nutzer/innen (Mao et al. 2012).

Insofern kann die eingangs gestellte Frage, die auf eine mögliche psychopathologische Komorbidität abzielt, nicht eindeutig beantwortet werden, zumal Ängste und Depressionen im Zusammenhang mit einer eingetretenen Krebserkrankung auch ohne Bezug zur KM nicht selten sind und nur im langfristigen Verlauf, d.h. im Vergleich zwischen der Vorerkrankungsphase und der Akutphase ermittelt werden kann, inwieweit affektive Störungen einen habituellen bzw. situativen Charakter haben (vgl. Vehling et al. 2012). Schließlich hängt es auch vom jeweils eingesetzten Methodenarsenal ab, inwieweit Zusammenhänge zwischen psychischer Komorbidität und vor, bei und nach einer Krebserkrankung ermittelt werden können (vgl. dazu Mehnert et al. 2012 für Deutschland).

Die Vielzahl von Gründen, warum KM gewählt oder nicht gewählt wird, lässt sich - nicht nur bei Krebserkrankungen - aufteilen in solche, die auf eine anzie- 
hende Wirkung hindeuten (Pull-Faktoren) und solche, die eine abstoßende Wirkung (der vorherigen oder gleichzeitigen konventionellen Behandlung) zum Inhalt haben (Push-Faktoren) (im Überblick Hoefert \& Walach 2013, speziell zu Prostatakrebs Boon et al. 2003, Porter et al. 2008). Die Entscheidung zugunsten einer bestimmten KM-Methode oder mehrerer Methoden gleichzeitig fällt selten nur auf der Grundlage eines einzelnen Faktors, sondern ist zumeist Ergebnis eines Abwägungsprozesses zwischen den jeweils individuell in Betracht gezogenen eigenen Überzeugungen und Informationen. Will man einen Patienten sachkundig beraten, dann sind zum einen dessen Prioritäten in Betracht zu ziehen, und zum anderen dessen Informationsbasis. So ist - für alle Krebsarten erkennbar, dass ein Teil der Patienten lediglich an einem besseren Management der eigenen Symptome und Nebenwirkungen der konventionellen Behandlung interessiert ist, während ein anderer Teil eine womöglich spirituelle Sinngebung im Zusammenhang mit dem KM-Gebrauch zu finden hofft.

Die entsprechenden Informationen über KM werden mehr oder weniger „aktiv“ (auch aus dem Internet) eingeholt und aufgrund des eigenen Laienstatus überprüft (vgl. Evans et al. 2007a) bzw. kommt die eigene Entscheidung für oder gegen KM mehr oder weniger durch Druck von Familienangehörigen zustande (vgl. Zeliadt et al. 2006). Dabei haben die familiären Erwartungen auch kulturell eine unterschiedlich große Bedeutung (für Japan Hirai et al. 2008). Schließlich scheint auch das Geschlecht eine Bedeutung zu haben: Der Wunsch, mit Hilfe von KM den Krankheitsprozess unter Kontrolle bringen und ihn steuern zu können, ist offenbar bei Männern stärker ausgeprägt (Hedderson et al. 2004). Jene haben offenbar einen stärkeren „pragmatischen“ Ansatz bzw. betrachten sich selbst eher als „Verbraucher“, indem sie Lücken in der konventionellen Versorgung zu schließen versuchen oder ein Produkt bei wahrgenommener Ineffektivität oder Schädlichkeit einfach abwählen (vgl. Evans et al. 2007b). Manche von ihnen entscheiden sich für KM, weil sie Impotenz oder Inkontinenz durch eine konventionelle Behandlung befürchten (Singh et al. 2005). Umgekehrt scheinen für Frauen eher die Sorgen um die eigene Gesundheit ausschlaggebender für den KMGebrauch (schon vor einer aktuellen Krebsdiagnose) zu sein (Strait \& Furnham 2012). Die Präferenz für KM wird von ihnen auch nicht allein mit dem Motiv der Krebsprävention (bei familiärer Vorbelastung) begründet, sondern aus allgemeineren Motiven (Field et al. 2009), eine Tendenz, die sich nach einer aktuellen Diagnose fortzusetzen scheint.

Die Gründe, sich gegen KM zu entscheiden, sind zumeist konkreter: Örtliche und zeitliche Zugangsbarrieren, Misstrauen auch wegen möglicher schädlicher Wechselwirkungen mit konventionellen Medikamenten, mangelnde wissenschaftliche Evidenz oder Zufriedenheit mit der konventionellen Behandlung (Beatty et al. 2012). Auch eine deutsche Studie zeigt durchaus Zufriedenheit mit der konventionellen Behandlung (Tautz et al. 2012). Dabei ist auffällig, dass sowohl Befürworter als auch Gegner von KM häufig zu einer Polarisierung (bei den KM-Befürwortern im Sinne von $\mathrm{KM}=$ sicher, natürlich, ganzheitlich wirkend, 
konventionell $=$ schädigend, unnatürlich, nur lokal wirkend) neigen, ohne dass die jeweils positiven Aspekte einer konventionellen Behandlung bzw. die möglichen negativen Aspekte einer KM-Behandlung (vgl. Tascilar et al. 2006) genügend in Betracht gezogen werden. Die jeweils genannten Gründe differieren nicht wesentlich zwischen den Krebsarten, die hier im Blickpunkt stehen. Die mehr oder weniger häufig genannten Gründe für den Gebrauch von KM bei Prostatakrebs haben Bishop et al. (2011) zusammengestellt (s. Tab. 14).

Tab. 14 Nutzungsmotive von KM bei Patienten mit Prostatakrebs (nach Bishop et al. 2011)

\begin{tabular}{|c|c|}
\hline Nutzungshäufigkeit & Nutzungsgründe \\
\hline $\begin{array}{l}\text { sehr häufig (in 50\% und mehr } \\
\text { der Fälle) }\end{array}$ & $\begin{array}{l}\text { den Krebs direkt bekämpfen } \\
\text { ein Rezidiv oder eine Ausbreitung verhindern } \\
\text { die eigene Lebenserwartung verlängern } \\
\text { die Lebensqualität verbessern } \\
\text { die Symptome generell mildern } \\
\text { mehr Kontrolle über die Genesung haben } \\
\text { Hoffnung gewinnen } \\
\text { das Immunsystem stärken } \\
\text { mit Stress besser umgehen können } \\
\text { Nebenwirkungen der konventionellen Therapie mildern }\end{array}$ \\
\hline häufig (in 25-50\% der Fälle) & $\begin{array}{l}\text { sich besser fühlen } \\
\text { die allgemeine Gesundheit verbessern } \\
\text { eine größere Kontrolle über den Krebs haben } \\
\text { psychologische Unterstützung erhalten } \\
\text { weil KM sehr sicher ist }\end{array}$ \\
\hline $\begin{array}{l}\text { weniger häufig (unter 25\% } \\
\text { der Fälle) }\end{array}$ & $\begin{array}{l}\text { wegen der medizinischen/wissenschaftlichen Information } \\
\text { weil Freunde gute Erfahrungen mit KM gemacht haben } \\
\text { um damit einmal zu experimentieren } \\
\text { auf Empfehlung des Arztes } \\
\text { wegen des gegenwärtig schlechten Gesundheitsstatus } \\
\text { wegen Unzufriedenheit mit der konventionellen Therapie } \\
\text { wegen Desinteresses der offiziellen Behandler } \\
\text { weil CAM wirksam ist } \\
\text { weil es hilft, mit Symptomen wie Schmerz oder Müdigkeit besser umzugehen } \\
\text { um einen ganzheitlicheren Ansatz auszuprobieren } \\
\text { um eine engere Beziehung zum Behandler zu gewinnen } \\
\text { weil die Familie und Freunde dazu drängen }\end{array}$ \\
\hline
\end{tabular}


In einer kleinen deutschen qualitativen Studie hatten Paul et al. (2013) als Gründe für den KM-Gebrauch vor allem „Erhaltung der eigenen Stärke“ und „Etwas für sich selbst tun können“ ermittelt. In der bereits oben erwähnten Literaturanalyse von Wanchai et al. (2010) über Motive bei Brustkrebs-Patientinnen standen als Motive für den KM-Gebrauch die Verbesserung der eigenen Gesundheit, die Stärkung des Immunsystems, die Reduzierung von Nebenwirkungen durch die konventionelle Behandlung an vorderster Stelle, daneben wurden als Motive (von den Frauen) aber auch die Verbesserung der emotionalen Gesundheit und die Verbesserung des Gefühls, die Dinge unter Kontrolle zu haben, genannt.

Insgesamt ist im Einzelfall jeweils zu beurteilen, welchen Stellenwert KM hat: KM kann gewählt werden aus dem Glauben an eine - womöglich krankheitsunspezifische - Wirksamkeit, als schlichtes Instrument, das sich in das Repertoire der anderen (meist konventionellen) Instrumente einreiht und wo bei mehreren KM-Alternativen die einzelne Effektivität im Vergleich bestimmt wird, als (mitunter symbolisches) Instrument, mit dessen Gebrauch man sich Hoffnung machen kann (vgl. Güthlin et al. 2010) oder schließlich als „letzte Chance“ (vgl. Verhoef et al. 2005), wobei die Gewissheit von „Aussichtslosigkeit“ in der Krebsbekämpfung nicht selten auch durch den behandelnden (konventionellen) Arzt verstärkt wird (Kristoffersen et al. 2009).

\subsection{KM-Therapien}

Wenn mehr als 50\% aller Krebs-Patienten Methoden und Produkte der KM zumindest ausprobieren, dann verbinden sie damit recht unterschiedliche Hoffnungen: Während einige nur „nichts versäumen“ wollen, aber nicht vollends davon überzeugt sind, wollen andere speziell die Begleitsymptome einer Radiooder Chemotherapie mildern, ohne damit unbedingt die weltanschaulichen Hintergründe der jeweiligen KM-Form zu teilen. Einigen anderen geht es weniger um spezifische Wirkungen (z.B. in der Schmerzbekämpfung), sondern um die Herstellung eines allgemeinen Wohlgefühls und damit um eine Verbesserung der Lebensqualität. Ein gemeinsamer Nenner scheint darin zu bestehen, dass sie diesen Prozess - anders als bei einer biomedizinischen Krebstherapie - aktiv mitgestalten und womöglich auch kontrollieren können (vgl. Münstedt et al. 2007). Schließlich hat ein Teil der Patienten die Hoffnung, den Krebs direkt mit Hilfe von KM bekämpfen zu können (vgl. dazu Ernst 20ogb). Die Besserungs- oder Überlebensaussichten verschlechtern sich jedoch dann, wenn sie dabei ausschließlich (alternativ) auf KM-Methoden setzen. Immerhin steigt die Nutzungsprävalenz von KM an, wenn ein Tumor auf chirurgische Weise nicht entfernbar erscheint (Schieman et al. 2009), wobei am häufigsten Nahrungsmittelzusätze (z.B. Vitamine und Mineralien) sowie Kräuter und Tees zur Anwendung kommen (vgl. Cassileth \& Deng 2004, Chang et al. 2011).

Sofern Patienten dem gleichermaßen spezifischen wie auch realistischen Anspruch folgen, einzelne Symptome zu behandeln, die im Zusammenhang mit der 
Krebs-Erkrankung bzw. durch die chemo- oder radiotherapeutische Behandlung auftreten, scheinen einige KM-Verfahren durchaus hilfreich zu sein (im Überblick Henß et al. 2010). Derzeit liegt eine sehr informative Übersicht über die bei einer Krebserkrankung infrage kommenden Methoden vor (Hübner 2012, 2013), wobei die Wirksamkeit der jeweiligen Methoden entscheidend davon abhängt, ob die jeweilige Methode, alternativ zur einer konventionellen Therapie eingesetzt wird und dadurch möglicherweise bestimmte Heilungschancen versäumt werden, oder ob sie komplementär und in Abstimmung mit einer parallelen konventionellen Therapie zur Anwendung kommt (Münstedt \& Hübner 2013).

Beispielsweise

- eignen sich mehrere Verfahren zur Reduktion von Stress (operationalisiert durch eine Verringerung der endokrinen Indizes), darunter vor allem Verfahren wie Achtsamkeitsmeditation und bestimmte Formen des Yoga, weiterhin Akupunktur zur Angstminderung, wobei diagnostisch unterschieden werden sollte zwischen einem allgemeinen, krankheitsbedingten Stress und einer Stresszustand, der z.B. durch eine anstehende Operation ausgelöst wird (im Überblick Chandwani et al. 2012)

- scheinen die Möglichkeiten für eine Reduzierung von krebsbedingtem Schmerz mit KM-Methoden nur begrenzt. Obwohl ein breites Arsenal an Methoden zur Verfügung steht (vgl. Cassileth et al. 2007), helfen die meisten Verfahren nur kurzfristig (Bardia et al. 2006), haben sich zumindest bei dieser Schmerzätiologie nicht eindeutig bewährt (auch nicht Akupunktur, vgl. Lee et al. 2005, Lu \& Rosenthal 2013) oder müssen in ihrer Wirkung relativiert werden vor dem Hintergrund der den Patienten auch unabhängig von der Krebserkrankung zur Verfügung stehenden Coping-Strategien (Cassileth \& Keefe 2010)

- $\quad$ sind die Nachweise zur Wirksamkeit bei chronischer Erschöpfung derzeit noch unzureichend (Sood et al. 2007, Alraeck et al. 2011), wobei hier körperliche Aktivität (z.B. Walking) sowie kognitiv-verhaltenstherapeutische Interventionen relativ erfolgversprechend sind (Mitchell 2010)

- eignen sich Verfahren zur Reduzierung von Schlaflosigkeit in unterschiedlicher Weise; einigermaßen bewährt haben sich Akupressur, Tai Chi und Yoga, etwas weniger Akupunktur, und am wenigsten Kräuter (vgl. Sarris \& Byrne 2011), wobei auch Verfahren wie Achtsamkeitsmeditation eine allerdings nur kurzfristig anhaltende Wirkung zeigen (Andersen et al. 2013)

- können enger definierte Symptome wie Brechreiz und Übelkeit bei Chemotherapie mit Hypnose (Richardson et al. 2007), bestimmte Hautprobleme bei Radiotherapie mit homöopathischen Mitteln (Kassab et al. 2010) oder können Appetitlosigkeit und Speiseaufnahmeprobleme mit diversen Tees behandelt werden.

Weitere Anregungen für den Gebrauch von KM-Methoden auch im Rahmen der Selbsthilfe, die sich aus pragmatischer Sicht bewährt haben, finden sich bei Dobos \& Kümmel (2011). 
Im Folgenden werden noch einmal zusammenfassend die wesentlichen CAMMethoden in Form von Review-Ergebnissen aus den letzten 10 Jahren vorgestellt, wobei zu berücksichtigen ist, dass die hier verglichenen Studien zum Teil recht unterschiedliche Designs haben, zum Teil auf objektiv messbaren physiologischen Parametern und zum Teil auf Fragebogen-Selbstaussagen beruhen. Die jeweiligen Reviewer bemängeln dabei durchgängig die zum Teil kleinen Studiengrößen oder die verwendeten Messinstrumente. Dies mag zum Teil darauf zurückzuführen sein, dass im Bereich der KM die methodischen Anforderungen an eine evidenzbasierte komplementärmedizinische Krebsforschung noch nicht hinreichend erfüllt werden (vgl. dazu Zachariae \& Johannessen 2011), zum Teil auch darauf, dass gerade bei Krebs - anders als bei partialisierten oder örtlich begrenzten Leiden - die gesamte Person des Patienten betroffen ist, die unterschiedlichen psychischen und physischen Effekte in einer vergleichsweise engeren Relation stehen und damit isolierte Messungen keine Beurteilung des Cesamtzustandes zulassen. Die folgende Tabelle 15 gibt einen Überblick über häufig verwendete oder empfohlene KM-Verfahren und deren Bewährung ohne Anspruch auf Vollständigkeit. Die jeweils genannten Verfahren genügen in unterschiedlichem Maße den üblichen Evidenz-Kriterien (vgl. dazu Beuth 2010).

Tab. 15 Review-Ergebnisse zu einzelnen KM-Methoden

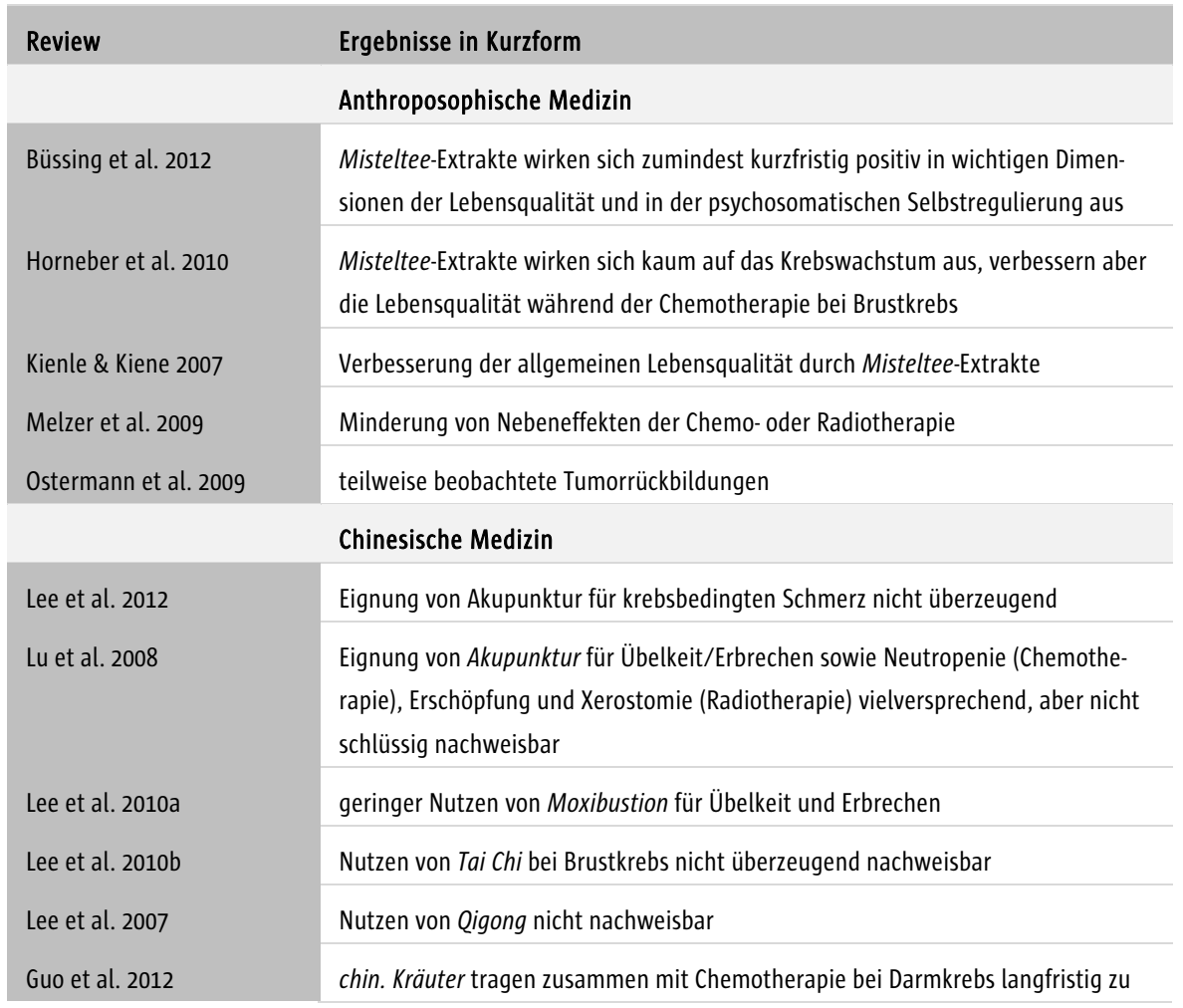




\begin{tabular}{|c|c|}
\hline Review & Ergebnisse in Kurzform \\
\hline \multirow{4}{*}{$\begin{array}{l}\text { Zhong et al. } 2012 \\
\text { Xu et al. } 2007\end{array}$} & positiven Effekten bei \\
\hline & Wirksamkeit von chin. Kräutern bei Darmkrebs schwer einschätzbar \\
\hline & Wirksamkeit von chin. Kräutern bei Schmerz nicht konsistent nachweisbar \\
\hline & Homöopathie \\
\hline \multirow{2}{*}{$\begin{array}{l}\text { Frenkel } 2010 \\
\text { Kassab et al. } 2010\end{array}$} & Effekte von Homöopathie bei Symptombesserung, Antitumor-Effekte fraglich \\
\hline & $\begin{array}{l}\text { deutliche Verbesserung von Radio- oder Chemotherapie-bedingten Nebenwirkun- } \\
\text { gen wie Dermatitis oder Stomatitis, nicht aber anderer Nebenwirkungen }\end{array}$ \\
\hline \multirow[t]{2}{*}{ Milazzo et al. 2006} & unzureichende Evidenz für die Wirksamkeit von Homöopathie bei Krebs \\
\hline & Mind-Body-Therapien \\
\hline Cramer et al. 2012a & kurzfristige Verbesserung des psychischen Wohlbefindens durch Yoga \\
\hline Zhang et al. 2012 & leichte Verbesserung von Lebensqualität-Werten durch Yoga \\
\hline Levine \& Balk 2012 & Verbesserung von Lebensqualität-Werten durch Yoga bei Brustkrebs \\
\hline Culos-Reed et al. 2012 & Verbesserung des Wohlgefühls bei Krebs-Überlebenden durch Yoga \\
\hline Smith \& Pukall 2009 & $\begin{array}{l}\text { unklar, welche Komponenten von Yoga wirken und bei welchen Patienten Yoga } \\
\text { wirksam ist }\end{array}$ \\
\hline Zainal et al. 2013 & $\begin{array}{l}\text { mittlere bis positive Evidenz dafür, dass Stress-Achtsamkeitsmeditation am mei- } \\
\text { sten zur Verringerung von Stress, danach Angst und danach Depression bei } \\
\text { Brustkrebs-Patientinnen beitragen kann }\end{array}$ \\
\hline Cramer et al. 2012b & $\begin{array}{l}\text { im Vergleich zur normalen Behandlung kann Stress-Achtsamkeitsmeditation Angst } \\
\text { und Depression verringern, führt aber nicht zu mehr Spiritualität bei Brustkrebs- } \\
\text { Patientinnen }\end{array}$ \\
\hline Musial et al. 2011 & $\begin{array}{l}\text { Stress-Achtsamkeitsmeditation scheint vor allem Werte der Lebensqualität und die } \\
\text { allgemeine Stimmung positiv zu beeinflussen, insgesamt noch nicht überzeu- } \\
\text { gend. }\end{array}$ \\
\hline \multirow[t]{2}{*}{ Ladesma \& Kumano 2009} & $\begin{array}{l}\text { Stress-Achtsamkeitsmeditation scheint die psychosoziale Anpassung an die } \\
\text { Krankheitssituation zu verbessern, Auswirkungen auf die physische Gesundheit } \\
\text { nicht überzeugend nachweisbar. }\end{array}$ \\
\hline & Körperzentrierte Methoden \\
\hline Fellows et al. 2004 & $\begin{array}{l}\text { Effekt von Aromatherapie-Massage auf Angst durchgängig nachweisbar, wenig auf } \\
\text { Depression und Schmerz }\end{array}$ \\
\hline $\begin{array}{l}\text { Yim et al. } 2009 \\
\text { Myers } 2008\end{array}$ & $\begin{array}{l}\text { Effekte von Aromatherapie-Massage auf Depression in einigen Studien nachweis- } \\
\text { bar }\end{array}$ \\
\hline Wilkinson et al. 2007 & $\begin{array}{l}\text { Effekte von Aromatherapie-Massage auf Angst und Depression bis zu zwei Wochen } \\
\text { nach Intervention, kurzfristige Wirkung auch bei Schmerz und Übelkeit }\end{array}$ \\
\hline
\end{tabular}




\begin{tabular}{|c|c|}
\hline Review & Ergebnisse in Kurzform \\
\hline Ernst 2009b & $\begin{array}{l}\text { Wirksamkeit von Massage bei Angst, Schmerz, Übelkeit, Erschöpfung, Ärger und } \\
\text { Stress tendenziell nachweisbar }\end{array}$ \\
\hline \multirow[t]{2}{*}{ Mishra et al. 2012} & $\begin{array}{l}\text { Verbesserung der körperlichen, psychischen und sozialen Funktionalität durch } \\
\text { Bewegungsprogramme, wobei die weniger herausfordernden effektiver sind als } \\
\text { die mittel bis stark herausfordernden Programme }\end{array}$ \\
\hline & Ernährung und Zusatzstoffe \\
\hline Hardy 2008 & $\begin{array}{l}\text { Eignung einer Reihe von Kräutern für Nebenwirkungen wie Stomatitis, Neuropa- } \\
\text { thie, Lymphedemie, Dermatitis oder Übelkeit }\end{array}$ \\
\hline Werneke et al. 2004 & $\begin{array}{l}\text { Nebenwirkungen (z.B. Blutungen) bei einigen Zusatzstoffen und Kräutern, dane- } \\
\text { ben gelegentlich negative Interaktionen mit Medikamenten }\end{array}$ \\
\hline Rhode et al. 2007 & Ingwer scheint das Zellwachstum bei Ovarialkrebs hemmen zu können \\
\hline Greenlee et al. 2012 & $\begin{array}{l}\text { zusätzliche Einnahme von Vitaminen } \mathrm{C} \text { und } \mathrm{E} \text { beeinflussen den Verlauf von Brust- } \\
\text { krebs positiv, Kombinations-Carotenoide negativ }\end{array}$ \\
\hline Stratton \& Godwin 2011 & zuätzliche Vitamin-Gaben beeinflussen nicht den Verlauf von Prostata-Krebs \\
\hline Ben-Arye et al. 2013 & $\begin{array}{l}\text { Wirksamkeit von Vitamin E, Glutamin oder Probiotika bei der Minderung von } \\
\text { Nebenwirkungen einer Radio- oder Chemotherapie }\end{array}$ \\
\hline Lippman et al. 2009 & $\begin{array}{l}\text { keine Senkung des Risikos für Prostata-Krebs und andere Krebsarten durch } \\
\text { Einnahme von Vitamin E und Selen }\end{array}$ \\
\hline Nechuta et al. 2011 & $\begin{array}{l}\text { Vitamineinnahme bis } 6 \text { Monate nach Brustkrebs-Diagnose verringert Mortalität } \\
\text { und Rezidiv-Wahrscheinlichkeit }\end{array}$ \\
\hline Shu et al. 2009 & Soja-haltige Ernährung verbessert Überlebenschancen bei Brustkrebs \\
\hline Dennert \& Horneber 2006 & $\begin{array}{l}\text { keine überzeugende Evidenz für die Wirkung von Selen bei der Milderung von } \\
\text { Nebeneffekten der Chemo- oder Radiotherapie }\end{array}$ \\
\hline van Patten et al. 2008 & $\begin{array}{l}\text { Studienlage nicht überzeugend, nach der durch Ernährung der negative Verlauf } \\
\text { von Prostatakrebs beeinflusst werden könnte }\end{array}$ \\
\hline & Spirituelle und künstlerische Methoden \\
\hline Schreiber \& Brockopp 2012 & $\begin{array}{l}\text { uneindeutige Beziehungen zwischen praktizierter Spiritualität/Religiosität und } \\
\text { psychischem Wohlbefinden bei überlebenden Brustkrebs-Patientinnen }\end{array}$ \\
\hline Ross et al. 2008 & Beten scheint die Anpassung an die Krankheitssituation verbessern zu können \\
\hline Peteet \& Balboni 2013 & $\begin{array}{l}\text { bessere Anpassung an die Krankheitssituation und geringerer Gebrauch aggressi- } \\
\text { ver Methoden im fortgeschrittenen Krebsstadium bei größerer Spiritualität }\end{array}$ \\
\hline Bradt et al. 2011 & $\begin{array}{l}\text { Wirkung von Musik-Interventionen auf Ängste und Stimmungen durch Senkung } \\
\text { der Atem- und Herzschlagfrequenz sowie des Blutdrucks }\end{array}$ \\
\hline
\end{tabular}


Das Arsenal der infrage kommenden KM-Therapien und Therapeutika ist damit keineswegs erschöpft. Sieht man einmal ab von eindeutig unseriösen Mitteln und Methoden, so verbleiben einige, bei denen eine bestimmte Grundvorstellung von Krebs vorliegt, die keineswegs irrational ist, für deren Operationalisierung aber die geeigneten Methoden und Mittel offenbar nur begrenzt geeignet sind:

- So gehen z.B. die Sauerstoff-Therapien (Sauerstoff-Ozontherapie, Sauerstoffmehrschritttherapie u.ä.) davon aus, dass sich Krebszellen besonders gut in sauerstoffarmen, schlecht durchbluteten Geweben vermehren können und Sauerstoffinhalation in Kombination mit Vitaminen und Mineralstoffen diesen Durchblutungsmangel beseitigen und damit das Krebswachstum beenden könne.

- Die Wärme-Therapien (Hyperthermie, Fiebertherapie u.ä.) lassen sich von der Vorstellung leiten, dass das Tumorgewebe besonders hitzeempfindlich sei und Krebszellen deshalb absterben können, wenn man sie einer bestimmten Temperatur aussetzen würde (dies wird mit Hilfe mit Hitzestrahlern, durch hochfrequente Ströme oder durch die künstliche Induktion von Fieber versucht). Auch die körpereigene Immunabwehr soll nach dieser Vorstellung bei guter Durchblutung besser funktionieren.

- Einigen weiteren Therapien liegt die Vorstellung zugrunde, dass Krebs durch eine Stärkung des Immunsystems bekämpft werden könne und entsprechend die Funktion der Thymusdrüse und der Milz durch Zuführung von ThymusExtrakten oder Peptiden gestärkt werden müsse (Thymustherapie). Auch wird aus einer anderen Sicht unterstellt, dass das Immunsystem die Krebszellen nicht immer erkenne, um sie wirksam bekämpfen zu können; entsprechend müssten die Krebszellen mit Hilfe von zugeführten Enzymen „demaskiert“ werden, um sie gezielt angreifen zu können (Enzymtherapie). Eine Variante stellt die Auffassung dar, dass die heutige Ernährung nicht ausgewogen und durch Vitamine und Mineralstoffe ergänzt werden müsse (Orthomolekulare Medizin).

- Auch gibt es die Auffassung, dass physiologische Prozesse, wie sie etwa durch biologisch orientierte Methoden (s.o.) direkt ausgelöst werden, auch indirekt in Gang gesetzt werden könnten, wie es die Vertreter geistiger Heilmethoden behaupten. Dazu zählen so unterschiedliche Methoden wie Handauflegen, Gesundbeten oder Fernbehandlung, deren Wirksamkeit zumindest nicht repräsentativ bestätigt werden konnte und deren Wirksamkeit in Einzelfällen zumeist auf Placebo-Effekte in der Interaktion mit dem Behandelnden oder auf Fremd- bzw. Autosuggestion zurückgeführt worden ist.

Sieht man einmal von der Frage der objektiven Wirksamkeit einer Methode, scheinen sich die Methoden auch in ihrer erlebten subjektiven Wirksamkeit zu unterscheiden: Gemessen am Kriterium des „benefit finding“ scheinen Energieheilen und verwandte Methoden in der persönlichen Wahrnehmung effektiver zu sein als Spezialdiäten, Phytopharmaka, Vitamine und Massage, während Akupunktur, 
Chiropraktik, Homöopathie, Entspannungsmethoden, Yoga und Tai Chi keinen Zuwachs an „benefit finding“ zeigen (Garland et al. 2013).

Es ist auch nicht auszuschließen, dass das jeweilige Ambiente, in das die Behandlung eingebettet ist (Station, interne und externe Krankenhausumgebung) einen zumindest indirekten Einfluss auf das Wohlbefinden und die Bereitschaft hat, an dem jeweiligen Ort eine Reihe von Maßnahmen zu erproben oder nicht.

\subsection{Psychoonkologie}

Die Psychoonkologie widmet sich einerseits der Erforschung von Zusammenhängen zwischen psychischen Dispositionen (Persönlichkeitsmerkmale, Formen der Stressbewältigung u.ä.) und der Krebsentstehung, andererseits aber auch der Frage, welche psychologischen und sonstigen Interventionen, $\mathrm{zu}$ denen auch eine Reihe von KM-Methoden (z.B. Meditation) zählen, geeignet sind, um das gestörte psychische Gleichgewicht im Anschluss an eine Krebsdiagnose und in der Folgezeit wieder herzustellen. Die erstgenannte Forschungsfrage kann insoweit beantwortet werden, als es keine „Krebspersönlichkeit“ zu geben scheint, mit deren Merkmalen man die Entstehung sehr unterschiedlicher Krebsformen oder auch die Verläufe und Prognosen der jeweiligen Erkrankungen erklären könnte (vgl. Tschuschke 2008).

\subsubsection{Psychische Prozesse}

Die Verwendung von KM während einer Krebserkrankung kann recht unterschiedliche psychische Prozesse in Gang setzen oder verstärken. So haben Smithson et al. (2010a) folgende Prozesse auf der Grundlage von PatientenInterviews aus verschiedenen Studien der Jahre 1998 bis 2007 identifizieren können:

- Kontrolle: Das Gefühl, die Kontrolle (wieder) zu gewinnen, war eher bei denjenigen vorhanden, die eine Methoden-Mixtur und Mind-Body-Methoden benutzten, seltener bei den Langzeit-Überlebenden und überhaupt nicht bei denjenigen mit fortgeschrittenem Krebs.

- Verbindungen: Viele KM-Nutzer/innen erleben (neue) soziale Verbindungen mit a) einem Therapeuten, b) mit einer (neuen) sozialen Gruppe und c) mit ihrem Körper oder ihrer Psyche, was sich zu a) besonders bei denjenigen zeigt, die Körpertherapien nutzen, sowie bei denjenigen mit fortgeschrittenem Krebs, nicht aber bei denjenigen mit Mind-Body-Therapien und den Langzeitüberlebenden zeigt. Verbindungen im Sinne von b) werden in allen Subgruppen erlebt, am stärksten bei den Nutzern von Körpertherapien. Das Verbindungserleben im Sinne von c) ist am stärksten ausgeprägt bei denjenigen mit einer Methoden-Mixtur und Mind-Body-Therapien, nicht aber bei den Nutzern von Körpertherapien und solchen mit fortgeschrittenem Krebs. 
- Wohlbefinden: Ein allgemeines Wohlbefinden stellt sich besonders bei denjenigen ein, die Körpertherapien verwenden, sowie bei denjenigen mit fortgeschrittenem Krebs.

- Transformation: Manche Patienten erleben langfristige Veränderungen in ihren Sichtweisen, ein Prozess, der vor allem bei denjenigen mit einer MethodenMixtur und den Nutzern von Mind-Body-Therapien zu beobachten ist.

- Polarisation: Manche Patienten erleben, dass es sich bei der KM und bei der Biomedizin um zwei gänzlich verschiedene Welten handelt. Dieser Prozess tritt besonders bei denjenigen auf, die eine Methoden-Mixtur und MindBody-Methoden nutzen, nicht aber bei den Nutzern von Körpertherapien und denjenigen mit fortgeschrittenem Krebs.

- Integration: Einige Patienten erleben die Integration von KM-Methoden in den regulären Behandlungsprozess; dieses Erleben ist, wenn es sich überhaupt einstellt, in allen Subgruppen etwa gleich häufig anzutreffen.

\subsubsection{Krankheitsvorstellungen bei Krebs}

Ein entscheidender Ansatzpunkt für die psychoonkologische Arbeit mit Patienten dürfte allerdings in den persönlichen „Theorien“ der Betroffenen von der Krebsentstehung und dem wahrscheinlichen Verlauf der Erkrankung liegen. $\mathrm{Zu}$ den - von den Patienten angenommenen - Ursachen zählen ebenso Genetik und Heredität wie auch Ernährung, körperliche Bewegung und Umgangsweisen mit Stress. Dazu kommt, dass man sich als Patient nicht nur mehr oder weniger verantwortlich für solche Ursachen fühlen kann, sondern dass sich daraus auch unterschiedliche emotionale Konsequenzen (Hadern mit dem Schicksal, Selbstvorwürfe, Schuldgefühle usw.) entwickeln können, und zwar unabhängig davon, ob diese Gründe tatsächlich objektiv medizinisch infrage kommen (vgl. Hoefert \& Brähler 2013).

Die jeweils als zutreffend angesehenen Gründe lassen sich auch danach unterscheiden, wieweit sie von den Patienten als kontrollierbar wahrgenommen werden; bei eher schicksalhaft erlebten Gründen ist das so gut wie nicht der Fall, bei falscher Ernährung, zu wenig Bewegung oder zu viel Exposition gegenüber belastenden Situationen dagegen in hohem Maße). So konnten Costanzo et al. (2011) in diesem Zusammenhang beispielsweise zeigen, dass überlebende BrustkrebsPatientinnen, die kontrollierbare Gründe annahmen, auch stärker bereit waren, nach Abschluss der rein medizinischen Behandlung Veränderungen in ihren Lebensgewohnheiten vorzunehmen. Wenn also eine KM-Methode aus Patientensicht geeignet erscheint, wieder Kontrolle über das eigene Leben bzw. die eigene Gesundheit zu erlangen, dann ist sie attraktiv für diejenigen, die von einer Ätiopathogenese durch eigenes Fehlverhalten in der Vergangenheit ausgehen; umgekehrt ist die Methode wenig attraktiv, wenn eine eher genetische, hereditäre oder schicksalshafte Verursachung angenommen wird. 


\subsubsection{Psychische Komorbidität}

Es ist nachvollziehbar, dass jemand unmittelbar nach einer Krebsdiagnose eine schwere Verunsicherung erlebt, die sich nicht nur auf die eigene Lebensperspektive, sondern auch auf berufliche und finanzielle Probleme erstreckt und bei der vor allem das weitere Schicksal der engeren Familienmitglieder einen zentralen Stellenwert hat. Als Reaktion auf diese neue Situation oder als Aktivierung prämorbider psychischer Probleme können Angststörungen, Depressionen und Verstimmungen auftreten bzw. aktualisiert werden (vgl. Mehnert et al. 2012). Im Rahmen einer sich anschließenden Chemotherapie können - meist vorübergehend - psychische Störungen wie Gedächtnis- und Konzentrationsschwierigkeiten auftreten (Joly et al. 2011). Hier stellt sich die Frage, ob KM-Nutzer/innen diesbezüglich eine auffällige Subgruppe bilden: So ist der Übersicht von Wanchai et al. (2010) der Hinweis zu entnehmen, dass zumindest Brustkrebs-Patientinnen mit einer Präferenz für KM häufiger über Symptome wie Schmerz, Steifheit, Benommenheit oder Schwellungen klagen als Patientinnen ohne diese Präferenz. Ein großer Teil der bei Wanchai et al. referierten Studien spricht außerdem für vergleichsweise höhere Angst- und Depressionswerte sowie anderer emotionaler Dysfunktionen bei KM-Nutzerinnen. Möglicherweise kann die KM-Nutzung (auch schon vor der Krebsdiagnose) auch als Versuch der psychologischen Selbstheilung verstanden werden, was sowohl durch KM-orientierte Mediziner als auch durch hinzugezogene Kollegen aus der Psychoonkologie abzuklären wäre.

\subsubsection{Psychoonkologische Beratung}

Bei der Bedarfseinschätzung von psychoonkologischer Beratung sollte erstens berücksichtigt werden, wieweit jemand bereits über ein stützendes soziales Netz verfügt, zweitens, welche Phasen sich als besonders sensibel erweisen, und drittens, welche Thematik in den jeweiligen Phasen im Vordergrund stehen sollte. So ist beispielsweise die Phase unmittelbar nach der Diagnose häufig durch Hoffnungs- und Hilflosigkeit, häufig auch durch Erfahrung von sozialer Isolation (andere Menschen meiden den Patienten, als habe er eine ansteckende Krankheit) gekennzeichnet. Eine weitere kritische Phase entsteht dann, wenn die medizinische Therapie beendet wird und damit die Struktur regelmäßiger Interventionen wegfällt und einer gewissen Struktur- und Perspektivlosigkeit für das weitere Leben Platz macht. Eine mögliche weitere kritische Phase entsteht dann, wenn trotz aller eigenen und fremden Bemühungen der Tod und das Sterben unausweichlich zu sein scheinen.

Solange dies noch nicht der Fall ist, dominieren in der Beratung zunächst Themen wie der Umgang mit dem Rollenverlust in der Familie und im Beruf, später dann - wenn therapie- oder krankheitsbedingte Einschränkungen auftreten Themen wie Sexualität, Ernährung, Verdauung und krankheitsbedingtem Stress oder Verstimmungen und Depressionen. Ansatzpunkte für eine gezielte Bera- 
tung sind dabei oft die Krankheitsvorstellungen, welche die Betroffenen von den Ursachen und dem wahrscheinlichen Verlauf der Krankheit haben und im ungünstigen Fall die Krankheitsverarbeitung sowie das alltägliche Leben beeinträchtigen und $\mathrm{zu}$ teilweise ungerechtfertigten Sorgen und Ängsten beitragen (im Überblick Hoefert \& Brähler 2013, bei Krebspatienten Tschuschke 2013).

\subsubsection{Arbeits- und Vernetzungsformen}

Die Psychoonkologie ist insbesondere in folgenden Phasen einer Krebserkrankung gefordert:

- nach der Diagnosemitteilung,

- im Rahmen der Behandlung und ihrer Nebenwirkungen,

- in der Rehabilitation und Nachsorge,

- bei Rezidiv und Auftreten von Metastasen sowie

- in der palliativen oder terminalen Phase (vgl. Heckl et al. 2011).

In einem Akutkrankenhaus kann sie mehr oder weniger in die Regelversorgung eingebunden sein, sofern die Zertifizierungsvoraussetzungen erfüllt sind. Bei dem Modell des Konsiliardienstes wird ein - in einer anderen Abteilung tätiger Psychoonkologe von einem medizinischen Onkologen hinzugezogen, beim Modell des Liaisondienstes nimmt der Psychoonkologe regelmäßig an Stationsbesprechungen und Visiten teil. Psychoonkologen nehmen nicht nur eine Beratungsfunktion für Patienten wahr (z.B. bei emotionalen Problemen oder Problemen in der Partnerschaft und im Beruf), sondern auch für Ärzte, wenn es um Fragen der Kooperation mit niedergelassenen Psychotherapeuten oder um die Planung von Reha-Maßnahmen geht. Sie sind zum Teil auch in der Lage, neuropsychologische Trainingsprogramme durchzuführen und bestimmte Methoden wie autogenes Training oder progressive Muskelentspannung zu vermitteln, die ebenfalls $\mathrm{zu}$ den KM-Methoden gezählt werden, aber von einem anderen theoretischen Hintergrund ausgehen als z.B. die meisten Mind-Body-Methoden oder gar spirituelle Methoden. Die „Medizinnähe“ der von der Psychoonkologie favorisierten Methoden erlaubt damit eine schnellere interfachliche Kommunikation und Integration in die medizinische Regelversorgung eines Krankenhauses.

\subsection{Arzt-Patient-Interaktion in der Onkologie}

Obwohl mindestens als die Hälfte der an Krebs Erkrankten von Methoden der KM Gebrauch macht oder dies beabsichtigt, finden entsprechende Diskussionen mit den betreuenden Onkologen nicht in entsprechender Quantität statt (Rausch et al. 2011). Eine solche Diskussion erscheint andererseits allein schon wegen möglicher Wechselwirkungen zwischen Medikamenten und KM-Produkten medizinisch notwendig (Davis et al. 2012). Auch aus Gründen der ärztlichen Sorgfalts- 
pflicht erscheint es somit angemessen, Fragen der Effektivität und Sicherheit von KM-Methoden, die entweder von Patienten praktiziert oder präferiert werden, oder die von Ärzten für empfehlenswert gehalten werden, im Hinblick auf eine einvernehmliche gemeinsame Entscheidungsfindung anzusprechen (Verhoef et al. 2008).

- So berichten Ge et al. (2013) darüber, dass eine Diskussion über KM mit Radiologen eher selten ist und dass hier besonders Frauen und Vollzeitbeschäftigte besonders zurückhaltend sind. Möglicherweise werden in dieser Behandlungsphase (Bestrahlung) die Onkologen eher als technische Fachspezialisten gesehen, denen gegenüber sich Patienten weniger öffnen wollen. Dazu kommt, dass manche Patienten ohnehin von der Wirksamkeit und Sicherheit „ihrer“ KM-Methode überzeugt sind und Ärzte generell nicht als die richtigen Gesprächspartner ansehen. Andere Patienten meinen, der KMGebrauch sei ihre „Privatsache“ und das Thema passe nicht in den Kontext eines ärztlichen Gesprächs (Davis et al. 2012). Generell scheinen Patienten zu unterscheiden zwischen Ärzten der konventionellen Medizin und KMSpezialisten, zu denen auch nicht-akademische Heilberufe zählen. Patienten stellen sich auf die vermuteten Erwartungen ihres jeweiligen Gegenübers ein und zeigen im einen Fall (konventioneller Arzt) ein eher rezeptives passives Rollenverhalten, im anderen Fall ein eher kollegiales und mitunter forderndes Rollenverhalten. Dazu kommt im zweitgenannten Fall, dass sie wie bei einer Reihe von Mind-Body-Methoden - mit den Behandlern häufig körperlich interagieren und nicht selten auch gemeinsame körperliche oder spirituelle Erfahrungen teilen (vgl. Hoefert 2011).

- Aufseiten der Patienten scheint die Gesprächsbereitschaft auch von soziodemografischen Merkmalen der Patienten abzuhängen: Jüngere und gebildetere Frauen zeigen eine größere Bereitschaft, über KM zu sprechen (Zhang et al. 2012); dies gilt auch für Krebs überlebende Patientinnen (Bennett et al. 2009). Umgekehrt fielen bei der Diskussion über Kräuter und Nahrungsmittelzusätze in der Studie von Kennedy et al. (2008) besonders Männer, jüngere Erwachsene und Angehörige von Minderheiten als besonders zurückhaltend auf.

- Weiterhin hängt die Gesprächsbereitschaft von der zur Diskussion stehenden KM-Methode ab; die Bereitschaft ist größer bei biologischen Methoden als bei Mind-Body-Methoden, wobei dann, wenn über biologische Methoden gesprochen wird, die entsprechenden Patienten auch zufriedener mit dem Gesprächsverlauf sind (Oh et al. 2010b). Es scheint so, als wenn die Gesprächsbereitschaft von Patienten bei (vermutlich) sozial akzeptierteren KMMethoden größer ist als bei (vermutlich) ungewöhnlichen Methoden und wenn aktuell von der Methode Gebrauch gemacht wird (Saxe et al. 2008). Außerdem akzeptieren Ärzte eher Methoden, die sie als unterstützend in ihrem konventionellen Vorgehen erleben als solche, die als „alternativ“ gelten 
und geeignet zu sein scheinen, einen Patienten zum Abbruch seiner konventionellen Therapie zu verleiten (vgl. Adams \& Jewell 2007, Hök et al. 2008).

- Patienten öffnen sich für KM-Themen auch in Abhängigkeit von der Gesprächsdynamik, wie die qualitative Studie von Shelley et al. (2009) mit niedergelassenen Ärzten zeigt: Die Gesprächsbereitschaft von Patienten ist größer, wenn der Gesprächspartner eine offene und vorurteilsfreie Haltung zeigt (wobei sie nicht unbedingt erwarten, dass der Arzt KM-Experte ist), wenn der Arzt von sich aus initiativ wird und die KM-Thematik anspricht (Patienten verhalten sich generell eher passiv und rezeptiv) und wenn der Arzt bei Fragen der Effektivität und Sicherheit sich nicht von vornherein auf sein wissenschaftliches Evidenzverständnis beruft und eine entsprechende Skepsis zum Ausdruck bringt. Häufige Unterbrechungen des Patienten durch den Arzt oder eine „belehrende“ Sprachweise scheinen wenig geeignet, die Gesprächsbereitschaft des Patienten zu fördern.

- Ärzte sind mehr oder weniger mit der jeweils zur Diskussion stehenden KMMethode vertraut und fragen oft nicht nach, weil sie glauben, zu wenig einschlägige Kenntnisse über KM zu haben (Zhang et al. 2012). Wenn Ärzte sich ihrer eigenen KM-Kenntnisse unsicher sind und dies auch zeigen, kann dies bei manchen Patienten zu Ängsten führen, die dann gelegentlich zu einem Therapieabbruch beitragen (Smithson et al. 2010). Generell sind Ärzte offenbar auch skeptischer bezüglich der Heilungschancen von Krebs oder der Chancen für die Stärkung des Immunsystems durch KM als die Patienten (Richardson et al. 2004), wobei manche Ärzte allerdings - für die Patienten oft überraschend - geradezu missionarisch bestimmte KM-Methoden propagieren und deshalb eine Skepsis auf der Patientenseite provozieren (vgl. Roberts et al. 2005).

- Man sollte davon ausgehen, dass Patienten und Ärzte nicht nur unterschiedliche Informationsquellen haben, aus denen sie ihre Kenntnisse über KrebsKrankheiten und angemessene Behandlungsmethoden beziehen, sondern auch unterschiedliche Vorstellungen vom Verlauf einer Krankheit (vgl. im Überblick Hoefert \& Brähler 2013). Ärzte beziehen sich zumeist auf Fachliteratur und in der eigenen Berufspraxis erlebte Krankheitsfälle aus Behandlersicht, Patienten dagegen auf Fälle im Bekanntenkreis sowie auf Informationen aus den öffentlich zugänglichen Medien einschließlich dem Internet, was nicht selten zu unbegründeten Ängsten einerseits oder zu unrealistischen Hoffnungen andererseits beiträgt (Broom et al. 20o8). Insofern können diverse Diskrepanzen in den Sichtweisen einer Krankheit und Einschätzungen der Wirksamkeit einer Methode entstehen. Diskrepante Sichtweisen beziehen sich aber nicht ausschließlich auf die KM, sondern auch darauf, was die konventionelle Krebsmedizin leisten kann und was nicht (neben Patienten, die zu viel von dieser Medizin erwarten, gibt es solche, die ihr sehr wenig zutrauen, vgl. O'Callaghan 2011). Derartige Diskrepanzen lassen sich oft durch eine angemessene Informationsvermittlung überbrücken; die Befürchtung 
mancher Ärzte, sie könnten ihre Patienten damit überfordern, scheint unbegründet (Lansdown et al. 2008).

- Die Bereitschaft von Krankenhaus-Ärzten, KM-Methoden kennen zu lernen, scheint dennoch relativ groß zu sein (Bjerså et al. 2012). Daneben erfordert, so vermuten viele durchaus an KM interessierte Ärzte, eine Diskussion über KM-Themen einen erhöhten Zeitbedarf im Krankenhaus-Ablauf, sodass eine Diskussion entweder nicht initiiert oder frühzeitig beendet wird. Wie viel Zeit jeweils erforderlich ist, hängt jedoch auch davon ab, wieweit die Diskussion über KM vom Arzt strukturiert oder didaktisiert wird, ohne damit den Patienten zu bevormunden, und wieweit gemeinsame Entscheidungen im Sinne eines shared decision making erlaubt oder initiiert werden (Schofield et al. 2010). Selbst dann, wenn Krankenhaus-Ärzte das KM-Thema von sich aus nicht oder unzureichend ansprechen, bleibt es dem Pflegepersonal vorbehalten, diesen Diskussionsmangel auszugleichen, da Pflegekräfte oft besser über KM-Methoden informiert sind als Ärzte (Broom \& Adams 2009).

Bei all dem darf nicht vergessen werden, dass die Krebsthematik für die meisten Patienten in hohem Maß angstbesetzt ist bzw. dass die Diskussion über KM diese Angst noch verstärken kann (Juraskova et al. 2010). Krebsängste bestehen bereits in der gesunden Bevölkerung, wobei die Erkrankung zumeist assoziiert wird mit unausweichlichem Tod und Siechtum (Hoefert 2012). Deshalb ist ein hohes Maß an Empathie - auch bei divergenten Einschätzungen der Wirksamkeit einer Methode - unabdingbar. Diese Auseinandersetzung mit den Verständnisweisen von Patienten erfordert eine gewisse Zeit, die Möglichkeit der Einbeziehung von anderen Fachspezialisten und eine möglichst stressarme Umgebung (allgemein dazu Verhoef et al. 2009, zu Prostatakrebs Klempner \& Bubley 2012, zu Brustkrebs Gerber et al. 2006), Bedingungen also, wie sie in manchen Fachkliniken bereits gegeben sind bzw. hergestellt werden könnten.

\subsection{Einige Implikationen für eine integrierte onkologische Versorgung im Krankenhaus}

Patienten, die bereit sind, sich auf eine KM-Methode einzulassen oder im Rahmen ihrer Krebserkrankung eine schon praktizierte Methode fortführen wollen, erleben nicht selten ein Wechselspiel zwischen Integration der verschiedenen Angebote innerhalb und außerhalb des Krankenhauses einerseits, und Polarisation auf professioneller und institutioneller Ebene andererseits. Im Falle der Integration sind die jeweiligen Angebote aufeinander abgestimmt, ergänzen sich weitgehend komplementär und werden sprachlich mit den gleichen Bedeutungsgehalten vermittelt. Im Falle der Polarisation sind die Angebote eher konkurrierend, schließen Alternativen aus und werden mit unterschiedlichen Bedeutungen und Wertungen vermittelt (vgl. dazu Smithson et al. 2010). Eine Integration der konventionellen und KM-Verfahren bietet sich allein schon deshalb an, weil 
manche Kombinationen zwischen beiden Verfahrensarten kontraindiziert sind, d.h. erhöhte Toxizitäten entstehen und Wirkungsminderungen bestimmter Verfahren eintreten können (Andersen et al. 2013). Eine Integration setzt auch voraus, dass Patienten und Ärzte über (womöglich parallelen) KM-Gebrauch der Patienten sprechen und dieser Gebrauch vorurteilsfrei und kenntnisreich diskutiert wird (vgl. Dobos et al. 2012 am Beispiel Brustkrebs).

Eine aus Sicht des Patienten kohärente Versorgung - einschließlich KM - setzt verschiedene Abstimmungsprozesse bereits krankenhausintern voraus. Denn idealerweise soll die „ganze Person“ des Patienten gewürdigt und behandelt werden (tatsächlich findet häufig eine institutionelle und professionelle Fraktionierung in körperliche und psychische Funktionen statt). Idealerweise sollen auch die beteiligten Professionen bei der Beratung eines Einzelfalls teamartig zusammenarbeiten oder zumindest als Team die wesentlichen Entscheidungen treffen (tatsächlich wird häufig nur delegiert oder an verschiedenen Orten isoliert entschieden). Insofern gibt es angesichts der hohen Prävalenz von Krebserkrankungen noch relativ wenige „Programme“, bei denen die jeweiligen Einzelmaßnahmen und Zuständigkeiten aufeinander abgestimmt sind. Seely et al. (2012) haben kürzlich derartige Programme - auch aus dem deutschen Sprachraum - untersucht und dabei auch festgestellt, welche KM-Methoden in solchen, krankenhausintern oder -extern wie häufig praktiziert werden: Auf den oberen Plätzen stehen demnach Mind-Body-Methoden, Massage, Ernährungsberatung, Kunsttherapie und Yoga. Gleichwohl werden aus dem internationalen Vergleich die Unterschiede deutlich, die sich aus den gesundheitspolitischen Rahmenbedingungen ergeben und sich auf die Finanzierung und Organisationsform (stationär, teilstationär, ambulant) auswirken. Unterschiede bestehen schließlich auch in dem Maße, in dem Angehörige in diese Programme einbezogen werden.

Trotz aller Komplexität einer „integrierten“ Versorgung lassen sich jedoch mehr oder weniger aufwändige Versorgungsformen unterscheiden. So kann z.B. auch ein Arzt mit relativ wenigen KM-Kenntnissen beurteilen, ob eine KM-Methode hilfreich sein kann bei der Minderung von Nebenwirkungen einer Chemo- oder Radiotherapie, insbesondere bei Symptomen wie Übelkeit, Schläfrigkeit oder Muskelschmerz; das Problem ist weitgehend durch Delegation lösbar. Anders stellt sich Lage dar, wenn offenbar eine psychische Komorbidität vorzuliegen scheint und entschieden werden muss, welche Art der Behandlung Vorrang hat. Aufwändiger wird die Beratung im Hinblick auf eine KM-Methode dann, wenn ein Verständnis des „philosophischen“ Hintergrundes erforderlich ist, um die Methode angemessen praktizieren zu können (z.B. bei der anthroposophischen Medizin oder Homöopathie) oder wenn eine Methode (z.B. Yoga oder Achtsamkeitsmeditation) nicht nur als bloße „Technik“ verstanden werden soll.

Häufig lässt sich Komplexität auch durch ein verbessertes Management reduzieren. Dieses beginnt bei der medialen und didaktisierten Gestaltung der Information für Patienten und erstreckt sich weiter auf die Kooperation mit hausinternen Abteilungen und externen Partnern, deren Dienste fallweise in Anspruch 
genommen werden können. Gerade bei Krebserkrankungen steht ein recht dichtes Netz von Institutionen und Spezialisten zur Verfügung ${ }^{15}$, in dem die KM weitgehend Akzeptanz gefunden hat bzw. die Chance hat, ihre Positionen und Angebote zu verbreiten. Die Bereitschaft von Ärzten mit eher konventioneller Orientierung, sich auf KM-Diskussionen einzulassen oder KM sogar zu empfehlen, dürfte nicht zuletzt davon abhängen, wieweit Studienergebnisse den KMGebrauch rechtfertigen: So sind beispielsweise die Fragen interessant, wieweit insbesondere biologische KM-Therapien mit konventioneller Radio- und Chemotherapie (und den dadurch ausgelösten biologischen Prozessen) verträglich sind, wie man sich die Effekte von KM-Therapien auf die gesundheitsbezogene Lebensqualität vorstellen kann und welchen vergleichsweisen Beitrag einzelne KMTherapien bei einer Krebserkrankung leisten (Robotin et al. 2012). Weiter bedarf es weiterer Untersuchungen zur Effektivität der Arzt-Patient-Interaktion in der Krebsberatung unter Einschluss der KM-Thematik, um so auch die Wirksamkeit konventioneller Therapien zu verbessern (Neumann et al. 2010). Und schließlich muss auch die institutionalisierte KM selbst sich bemühen, sich eventuell von bestimmten KM-Formen zu distanzieren, welche sich zumindest bei Krebserkrankungen als unwirksam erwiesen haben und sich womöglich nur die weit verbreitete Krebsangst ökonomisch nutzbar machen (vgl. Cassileth et al. 2012).

\section{Zusammenfassung}

Mehr als die Hälfte aller Krebspatienten nutzt nach einer Krebsdiagnose Methoden der komplementär-alternativen Medizin (KM) zumeist parallel zu einer konventionellen Behandlung und häufig ohne Wissen des behandelnden Arztes. Ein Teil der Nutzungsmotive ist im engeren Sinne symptombezogen, während ein anderer Teil der Verbesserung der gesundheitlichen und allgemeinen Lebensqualität gilt. Die Empirie zeigt, dass einige solcher Methoden durchaus geeignet sind, insbesondere solche Symptome, wie sie bei einer Radio- oder Chemotherapie auftreten, zu mildern. Verschiedene andere Methoden scheinen geeignet, das allgemeine Lebensgefühl zu stärken bzw. wiederherzustellen. Eine der Hauptaufgaben onkologischer Beratung besteht deshalb darin, über Nutzen und mögliche Nebenwirkungen von KM-Methoden aufzuklären und vor dem Hintergrund der individuellen Patientenmotivation die jeweils angemessene Anwendung zu finden.

\section{Literatur}

Adams, M., Jewell, A.P. (2007). The use of complementary and alternative medicine by cancer patients. Int Sem Surg Oncol, 4:10.

Alraek, T., Lee, M.S., Choi, T.-Y., Cao, H., Liu, J. (2011). Complementary and alternative medicine for patients with chronic fatigue syndrome: A systematic review. BMC Complement Altern Med, 11:87.

\footnotetext{
${ }^{15} \mathrm{Vgl}$. die Materialien der Deutschen Krebsgesellschaft (DKG) oder regionaler Krebsgesellschaften.
} 
Andersen, M.R., Sweet, E., Lowe, K.A., Standish, L.J., Drescher, C.W., Goff, B.A. (2013). Dangerous combinations: Ingestible CAM supplement use during chemotherapy in patients with ovarian cancer. I Altern Complement Med, 19(8), 714-720.

Andersen, S.R., Würtzen, H., Steding-Jessen, M., Christensen, I., Andersen, K.K., Flyger, H., Mitchelmore, C., Johansen, C., Dalton, S.O. (2013). Effect of mindfulness-based stress reduction on sleep quality: Results of a randomized trial among Danish breast cancer patients. Acta Oncologica, 52, 336-344.

Bardia, A., Barton, D.L., Prokop, L.J., Bauer, B.A., Moynihan, T.J. (2006). Efficacy of complementary and alternative medicine therapies in relieving cancer pain: A systematic review. I Clin Oncol, 24(34), 5457-5464.

Beatty, L., Koczwara, B., Knott, V., Wade, T. (2012). Why people choose to not use complementary therapies during cancer treatment: a focus group study. Eur J Cancer Care, 21(1), 98-106.

Bellizzi, K.M., Blank, T.0. (2007). Cancer-related identity and positive affect in survivors of prostate cancer. I Cancer Surv, 1(1), 44-48.

Ben-Arye, E., Pollack, A., Schiff, E., Tadmor, T., Samuels, N. (2013). Advising patients on the use of non-herbal nutritional supplements during cancer therapy: A need for doctor-patient-communication. I Pain Symptom Mange, i.Dr.

Bennett, J.A., Cameron, L.D., Whitehead, L.C., Porter, D. (2009). Differences between older and younger cancer survivors in seeking cancer information and using complementary/alternative medicine. I Gen Intern Med, 24(10), 1089-1094.

Beuth, J. (2010). Evidence-based complementary oncology: Innovative approaches to optimize standard therapy strategies. Anticancer Res, 30, 1767-1772.

Bishop, F.L., Lewith, G.T. (2010). Who uses CAM? A narrative review of demographic characteristics and health factors associated with CAM use. Evid Based Complement Alternat Med, 7(1), 11-28.

Bishop, F.L., Rea, A., Lewith, H., Chan, Y.K., Saville, I., Prescott, P., von Elm, E., Lewith, G.T. (2011). Complementary medicine use by men with prostate cancer: a systematic review of prevalence studies. Prostate Cancer Prostatic Dis, 14(1), 1-13.

Bjerså, K., Stener Victorin, E., Fagevik Olsen, M. (2012). Knowledge about complementary, alternative and integrative medicine (CAM) among registered health care providers in Swedish surgical care: a national survey among university hospitals. BMC Complement Altern Med, 12:42.

Boon, H., Brown, J.B., Gavin, A., Westlake, K. (2003). Men with prostate cancer: making decisions about complementary/alternative medicine. Med Decis Making, 23(6), 471-479.

Boon, H.S., Olatunde, F., Zick, S.M. (2007) Trends in comoplementary/alternative medicine use by breast cancer survivors: Comparing survey data from 1998 and 2005. BMC Womens Health, 7:4.

Bradt, I., Dileo, C., Grocke, D., Magill, L. (2011). Music interventions for improving psychological and physical outcomes in cancer patients. Cochrane Database Syst Rev, 8, CD006911.

Broom, A., Adams, I. (2009). Oncology clinicians' accounts of discussing complementary and alternative medicine with their patients. Health, 13(3), 317-336.

Broom, A., Tovey, P. (2008). The role of the Internet in cancer patients' engagement with complementary and alternative treatments. Health, 12(2), 139-155.

Büssing, A., Raak, C., Ostermann, T. (2012). Quality of life and related dimensions in cancer patients treated with mistletoe extract (iscador): a meta-analysis. Evid Based Complement Alternat Med, ID 219402.

Butler, S., Owen-Smith, A., Dilorio, C., Goodman, M., Liff, I., Steenland, K. (2011). Use of complementary and alternative medicine among men with prostate cancer in a rural setting. I Community Health, 36(6), 10041010.

Carpenter, C.L., Ganz, B.A., Bernstein, L. (2009). Complementary and alternative therapies among very long-term breast cancer survivors. Breast Cancer Res Treat, 116(2), 387-396.

Casellas-Grau, A., Font, A., Vives, I. (2013). Positive psychology interventions in breast cancer. A systematic review. Psychooncology, doi: 10.1002/pon.3353.

Cassileth, B.R., Yarett, I.R. (2012). Cancer quackery: The persistent popularity of useless, irrational alternative treatments. Oncology, 26(8), 754-758.

Cassileth, B., Keefe, F.J. (2010). Integrative and behavioural approaches to the treatment of cancer-related neuropathic pain. Oncologist, 15(suppl 2), 19-23.

Cassileth, B.R., Deng, G. (2004). Complementary and alternative therapies for cancer. Oncologist, 9, 80-89.

Cassileth, B., Trevisan, C., Gubili, J. (2007). Complementary therapies for cancer pain. Curr Pain Headache Rep, 11, $265-269$. 
Chan, J.M., Elkin, E.P., Silva, S.J., Broering, I.M., Latini, D.M., Carroll, P.R. (2005). Total and specific complementary and alternative medicine use in a large cohort of men with prostate cancer. Urology, 66(6), 1223-1228.

Chandwani, K.D., Ryan, J.I., Pappone, L.J., Janelsins, M.M., Sprod, L.K., Devine, K., Trevino, L., Gewandter, J., Morrow, G.R., Mustian, K.M. (2012). Cancer-related stress and complementary and alternative medicine: A review. Evid-based Complement Altern Med, ID979213.

Chang, K.H., Brodie, R., Choong, M.A., Sweeney, K.J., Kerin, M.J. (2011). Complementary and alternative medicine use in oncology: A questionnaire survey of patients and health care professionals. BMC Cancer, 11:96.

Costanzo, E.S., Lutgendorf, S.K., Roeder, S.L. (2011). Common-sense beliefs about cancer and health practices among women completing treatment for breast cancer. Psychooncology, 20(1), 53-61.

Cramer, H., Lange, S., Klose, P., Paul, A., Dobos, G. (2012a). Yoga for breast cancer patients and survivors: a systematic review and meta-analysis. BMC Cancer, 12:412.

Cramer, H., Lauche, R., Paul, A., Dobos, G. (2012b). Mindfulness-based stress reduction for breast cancer - a systematic review and meta-analysis. Curr Oncol, 19(5), e343-e352.

Culos-Reed, S.N., Mackenzie, M.J., Sohl, S.J., Jesse, M.T., Zahavich, A.N., Danhauer, S.C. (2012). Yoga \& cancer interventions: a review of the clinical significance of patient reported outcomes for cancer survivors. Evid Based Complement Alternat Med, Art. CD642576.

Damery, S., Gratus, C., Grieve, R., Warmington, S., Jones, I., Routledge, P., Greenfield, S., Dowswell, G., Sherriff, J., Wilson, S. (2011). The use of herbal medicines by people with cancer: a cross-sectional survey. $\mathrm{Br}$ I Cancer, 104(6), 927-933.

Davidson, R., Geogeghan, L., McLaughlin, L., Woodward, R. (2004). Psychological characteristics of cancer patients who use complementary therapies. Psycho-Oncology, 14(3), 187-195.

Davis, E.L., Oh, B., Butow, P.N., Mullan, B.A., Clarke, S. (2012). Cancer patient disclosure and patient-doctorcommunication of complementary and alternative medicine use: A systematic review. Oncologist, 17(11), $1475-1481$.

Dennert, G., Horneber, M. (2006). Selenium for alleviating the side effects of chemotherapy, radiotherapy and surgery in cancer patients. Cochrane Database Syst Rev, 3, ID005037.

Dobos, G., Kümmel, S. (2013). Gemeinsam gegen den Krebs. München (Zabert Sandmann).

Dobos, G.J., Voiss, P., Schwidde, I., Choi, K.-E., Paul, A., Kirschbaum, B., Saha, F.J., Kümmel, S. (2012). Integrative oncology for breast cancer patients: introduction of an expert-based model. BMC Cancer, 12:539.

Eng, I., Ramsum, D., Verhoef, M., Guns, E., Davison, I., Gallagher, R. (2003). A population-based survey of complementary and alternative medicine use in men recently diagnosed with prostate cancer. Integr Cancer Ther, 2(3), 212-216.

Epplein, M., Zheng, Y., Zheng, W., Chen, Z., Gu, K., Penson, D., Lu, W., Shu, X.-0. (2011). Quality of life after breast cancer diagnosis and survival. I Clin Oncol, 29(4), 406-412.

Ernst, E. (2009a). How much of CAM is based on research evidence? eCAM, 1-3, doi: 10.1093/ecam/nep044.

Ernst, E. (2009b). Complementary and alternative medicine (CAM) and cancer: the kind face of complementary medicine. Int I Surg, 7(6), 499-500.

Ernst, E. (2009c). Massage therapy for cancer palliation and supportive care: a systematic review of randomized clinical trials. Support Care Cancer, 17(4), 333-337.

Ernst, E., Schmidt, K., Steuer-Vogt, M.K. (2003). Mistletoe for cancer? A systematic review of randomised clinical trials. Int I Cancer, 107, 263-267.

Evans, M.A., Shaw, A., Thompson, E.A., Falk, S., Turton, P., Thompson, T., Sharp, D. (2007a). Decisions to use complementary and alternative medicine (CAM) by male cancer patients: information-seeking roles and types of evidence used. BMC Complement Altern Ther, 7:25.

Evans, M.A., Shaw, A., Sharp, D.J., Thompson, E.A., Falk, S., Turton, P., Thompson, T. (2007b). Men with cancer: is their use of complementary and alternative medicine a response to needs unmet by conventional care? Eur I Cancer Care, 16, 517-525.

Fellowes, D., Barnes, K., Wilkinson, S. (2004). Aromatherapy and massage for symptom relief in patients with cancer. Cochrane Database Syst Rev, 2, CD002287.

Field, K.M., Jenkins, M.A., Friedlander, M.L., McKinley, J.M., Price, M.A., Weideman, P., Keogh, L.A., McLachlan, S.A., Lindeman, G.J., kConFab Investigators, Hopper, J.L., Butow, P.N., Phillips, K.A. (2009). Predictors of the use of complementary and alternative medicine (CAM) by women at high risk for breast cancer. Eur I Cancer, 45(4), 5551-560.

Frenkel, M. (2010). Homeopathy in cancer care. Altern Ther Health Med, 16(3), 12-16. 
Gansler, T., Kaw, C., Crammer, C., Smith, T. (2008). A population-based study of prevalence of complementary methods use by cancer survivors. Cancer, 113(5), 1048-1057.

Garland, S.N., Valentine, D., Desai, K., Li, S., Langer, C., Evans, T., Mao, I.J. (2013). Complementary and alternative medicine (CAM) use and benefit finding among cancer patients. I Altern Complement Med, i. Dr.

Ge, J., Fishman, J., Vapivala, N., Li, S.Q., Desai, K., Xie, S.X., Mao, I.J. (2013). Ptient-physician communication about complementary and alternative medicine in a radiation oncology setting. Int / Radiat Oncol Biol Phys, 85(1), e1-6.

Gerber, B., Scholz, C., Reimer, T., Briese, V., Janni, W. (2006). Complementary and alternative therapeutic approaches in patients with early breast cancer: a systematic review. Breast Cancer Res Treat, 95, 199-209.

Gottschling, S., Meyer, S., Längler, A., Scharifi, G., Ebinger, F., Gronwald, B. (2013). Differences in use of complementary and alternative medicine between children and adolescents with cancer in Germany: A population based survey. Pediatr Blood Cancer, i.Dr.

Greenlee, H., Kwan, M.L., Ergas, I.J., Sherman, K.J., Krathwohl, S.E., Bonnell, C., Lee, M.M., Kushi, L.H. (2009). Complementary and alternative therapy before and after breast cancer diagnosis: The Pathways Study. Breast Cancer Res Treat, 117(3), 653-665.

Greenlee, H., Kwan, M.L., Kushi, L.H., Song, I., Castillo, A., Weltzien, E., Quesenberry, C.P. jr., Caan, B.J. (2012). Antioxidant supplement use after breast cancer diagnosis and mortality in the Life After Cancer Epidemiology (LACE) cohort. Cancer, 118(8), 2048-2058.

Güthlin, C., Walach, H., Naumann, I., Bartsch, H.-H., Rostock, M. (2010). Characteristics of cancer patients using homeopathy compared with those in conventional care: a cross-sectional study. Ann Oncol, 21, 1094-1099.

Guo, Z., Jia, X., Liu, J.P., Liao, J., Yang, Y. (2012). Herbal medicines for advanced colorectal cancer. Cochrane Database Syst Rev, 5, CD004653.

Haeck, M., Franke, S., Wiskemann, I., Mathiak, K. (2012). Psychoonkologie. Nervenarzt, 83, 1468-1476.

Hardy, M.L. (2008). Dietary supplement use in cancer care: Help or harm. Hematol Oncol Clin N Am, 22, 581-617.

Heckl, U., Singer, S., Wickert, M., Weis, J. (2011). Aktuelle Versorgungsstrukturen in der Psychoonkologie. Nervenheilkunde, 3, 124-130.

Hedderson, M.M., Patterson, R.E., Neuhouser, M.L., Schwartz, S.M., Bowen, D.J., Standish, L.J., Marshall, L.M. (2004). Sex differences in motives for use of complementary and alternative medicine among cancer patients. Altern Ther Health, 10(5), 58-64.

Helyer, L.K., Chin, S., Chui, B.K., Fitzgerald, B., Verma, S., Rakovitch, E., Dranisaris, G., Clemons, M. (2006). The use of complementary and alternative medicines among patients with locally advanced breast cancer - a descriptive study. BMC Cancer, 6:39.

Henß, H., Münstedt, K., Jückstock, J., Reinert, E., Hübner, J. (2010). Supportive Maßnahmen der Komplementärmedizin. Onkologe, 16(8), 795-801.

Hirai, K., Komura, K., Tokoro, A., Kuromaru, T., Ohshima, A., Ito, T., Sumiyoshi, Y., Hyodo, I. (2008). Psychological and behavioral mechanisms influencing the use of complementary and alternative medicine (CAM) in cancer patients. Ann Oncol, 19, 49-55.

Hoefert, H.-W. (2011). Patienten zwischen konventioneller und komplementär-alternativer Medizin. In: Hoefert, H.W., Klotter, C.(Hrsg.). Wandel der Patientenrolle. Neue Interaktionsformen im Gesundheitswesen. Göttingen (Hogrefe), 217-234.

Hoefert, H.-W. (2012). Angst vor Krebs. In: Hoefert, H.-W., Klotter, S. (Hrsg.). Gesundheitsängste. Lengerich (Pabst), 112-129.

Hoefert, H.-W. (2013). Patienten mit Präferenz für die komplementär-alternative Medizin. In: Hoefert, H.-W., Härter, M. (Hrsg.). Schwierige Patienten. Bern (Huber), 77-95.

Hoefert, H.-W., Brähler, E. (Hrsg.) (2013). Krankheitsvorstellungen von Patienten - Herausforderung für Medizin und Psychotherapie. Lengerich (Pabst).

Hoefert, H.-W., Walach, H. (2013). Krankheitsvorstellungen und Motivationen bei Nutzern der komplementäralternativen Medizin. In: Hoefert, H.-W., Brähler, E. (Hrsg.). Krankheitsvorstellungen von Patienten - Herausforderung für Medizin und Psychotherapie. Lengerich (Pabst), 279-306.

Hök, J., Tishelman, C., Ploner, A., Forss, A., Falkenberg, T. (2008). BMC Complement Altern Med, 8:48.

Horneber, M., Büschel, G., Dennert, G., Less, D., Ritter, E., Zwahlen, M. (2012). How many cancer patients use complementary and alternative medicine: a systematic review and meta-analysis. Integr Cancer Ther, 11(3), 187-203.

Horneber, M.A., Büschel, G., Huber, R., Linde, K., Rostock, M. (2010). Mistletoe therapy in oncology. Cochrane Database Syst Rev, 2, CD003297. 
Hübner, I. (2013). Komplementäre und Alternative Medizin in der Onkologie (II). Versicherungsmedizin, 65(3), 136-139.

Hübner, J. (2012). Komplementäre und Alternative Medizin in der Onkologie (I). Versicherungsmedizin, 65(2), 7983.

Joly, F., Rigal, 0., Noal, S., Giffard, B. (2011). Cognitive dysfunction and cancer: which consequences in terms of disease management? Psychooncology, 20(12), 1251-1258.

Juraskova, I., Hegedus, L., Butow, P., Smith, A., Schofield, P. (2010). Discussing complementary therapy use with early-stage breast cancer patients: exploring the communication gap. Integr Cancer Ther, 9(2), 168-176.

Kassab, S., Cummings, M., Berkovitz, S., van Haselen, R., Fisher, P. (2010). Homeopathic medicines for adverse effects of cancer treatments. Cochrane Database Syst Rev, CD004845.

Kennedy, I., Wang, C.C., Wu, C.H. (2008). Patient disclosure about herb and supplement use among adults in the US. Evid Based Complement Alternat Med, 5(4), 451-456.

Kienle, G.S., Kiene, H. (2007). Complementary cancer therapy: A systematic review of prospective clinical trials on anthroposophic mistletoe extracts. Eur | Med Res, 12, 103-119.

Kienle, GG.S., Glockmann, A., Schink, M., Keine, H. (2009). Viscum album L. extracts in breast and gynaecological cancers: a systematic review of clinical and preclinical research. J Exp Clin Cancer Res, 28:79.

Klempner, S.J., Bubley, G. (2012). Complementary and alternative medicines in prostate cancer: From bench to bedside? Oncologist, 17(6), 830-837.

Kristoffersen, A.E., Fønnebø, V.M., Norheim, A.J. (2009). Do cancer patients with a poor prognosis use complementary and alternative medicine more often than others? | Altern Complement Med, 15(1), 35-40.

Kristoffersen, A.E., Norheim, A.J., Fønnebø, V.M. (2012). Any difference? Use of a CAM provider among cancer patients, coronary heart disease (CHD) patients and individuals with no cancer/CHD. BMC Complement Altern Med, 12:1.

$\mathrm{Ku}, \mathrm{C.F} ., \mathrm{Koo}, \mathrm{M}$. (2012). Association of distress symptoms and use of complementary medicine among patients with cancer. I Clin Nurs, 21(5-6), 736-744.

Kushi, L.H., Kwan, M.L., Lee, M.M., Ambrosone, C.B. (2007). Lifestyle factors and survival in women with breast cancer. I Nutr, 137, 236-242.

Ladesma, D., Kumano, H. (2009). Mindfulness-based stress reduction and cancer: a meta-analysis. Psychooncology, 18(6), 571-579.

Lansdown, M., Martin, L., Fallowfield, L. (2008). Patient-physician interactions during early breast-cancer treatment: results from an international online survey. Curr Med Res Opin, 24(7), 1891-1904.

Lee, H., Schmidt, K., Ernst, E. (2005). Acupuncture for the relief of cancer-related pain - a systematic review. Eur ) Pain, 9(4), 437-444.

Lee, H., Schmidt, K., Ernst, E. (2012). Acupuncture for the relief of cancer-related pain - a systematic review. Eur ] Pain, 9:437.

Lee, M.S., Chen, K.W., Sancier, K.M., Ernst, E. (2007). Qigong for cancer treatment: A systematic review of controlled clinical trials. Acta Oncologica, 46, 717-722.

Lee, M.S., Choi, T.-Y., Park, J.-E., Lee, S.-S., Ernst, E. (2010a). Moxibustion for cancer care: a systematic review and meta-analysis. BMC Cancer, 10:130.

Lee, M.S., Choi, T.Y., Ernst, E. (2010b). Tai Chi for breast cancer patients: a systematic review. Breast Cancer Res, 120(2), 309-316.

Levine, A.S., Balk, J.L. (2012). Yoga and quality-of-life improvement in patients with breast cancer: a literature review. Int I Yoga, 22, 95-99.

Lippman, S.M., SELECT (2009). Effect of selenium and vitamin E on risk of prostate cancer and other cancers. IAMA, 301(1), 39-51.

Lu, W., Dean-Clower, E., Doherty-Gilman, A., Rosenthal, D.S. (2008). The value of acupuncture in cancer care. Hematol Oncol Clin North Am, 22(4), 631.

Lu, W., Rosenthal, D.S. (2013). Acupuncture for cancer pain and related symptoms. Curr Pain Headache Rep, 17(3), 1-8.

Ma, H., Carpenter, C.L., Sullivan-Halley, I., Bernstein, L. (2011). The role of herbal remedies in survival and quality of life among long-term breast cancer survivors - results of a prospective study. BMC Cancer, 11:222.

Mao, I.J., Farrar, I.T., Xie, S.X., Bowman, M.A., Armstrong, K. (2007). Use of complementary and alternative medicine and prayer among a national sample of cancer survivors compared to other populations without cancer. Complement Ther Med, 15(1), 21-29. 
Mao, I.J., Cronholm, P.F., Stein, E., Straton, J.B., Palmer, S.C., Barg, F.K. (2010). Positive changes, increased spiritual importance, and complementary and alternative medicine (CAM) use among cancer survivors. Integr Cancer Ther, 9(4), 339-347.

Mao, I.J., Palmer, C.S., Healy, K.E., Desai, K., Amsterdam, J. (2011). Complementary and alternative medicine use among cancer survivors: a population-based study. I Cancer Surv, 5(1), 8-17.

Mao, I.J., Palmer, S.C., Desai, K., Li, S.Q., Armstrong, K., Xie, S.X. (2012). Development and validation of an instrument for measuring attitudes and beliefs about complementary and alternative medicine (CAM) use among cancer patients. Evid Based Complement Altern Med, ID 798098.

Matsuno, R.K., Pagano, I.S., Maskarinec, G., Issell, B.F., Gotay, C.C. (2012). Complementary and alternative medicine use and breast cancer prognosis: a pooled analysis of four population-based studies of breast cancer survivors. I Womens Health, 21(12), 1252-1258.

Matthews, A.K., Sellergren, S.A., Huo, D., List, M., Fleming, G. (2007). Complementary and alternative medicine usw among breast cancer survivors. J Altern Complement Med, 13(5), 555-562.

McDermott, C.L., Blough, D.K., Fedorenko, C.R., Arora, N.K., Zeliadt, S.B., Fairweather, M.E., Oakley-Girvan, I., van den Eeden, S.K., Ramsey, S.D. (2012). Complementary and alternative medicine use among newly diagnosed prostate cancer patients. Support Care Cancer, 20(1), 65-73.

Mehnert, A., Koch, U., Schulz, H., Wegscheider, K., Weis, J., Faller, H., Brähler, E., Härter, M. (2012). Prevalence of mental disorders, psychosocial distress and need for psychosocial support in cancer patients - a study protocol of an epidemiological multi-center study. BMC Psychiatry, 12:70.

Melzer, J., Iten, F., Hostanska, K., Saller, R. (2009). Efficacy and safety of mistletoe preparations (Viscum album) for patients with cancer diseases. A systematic review. Forsch Komplementmed, 16(4), 217-226.

Michell, S.A. (2010). Cancer-related fatigue: state of the science. PM R, 2(5), 364-383.

Milazzo, S., Russell, N., Ernst, E. (2006). Efficacy of homeopathic therapy in cancer treatment. Eur J Cancer, 42(2), 282-289.

Mishra, S.I., Scherer, R.W., Snyder, C., Geigle, P.M., Berlanstein, D.R., Topaloglu, 0. (2012). Exercise interventions on health-related quality of life for people with cancer during active treatment. Cochrane Database Syst Rev, 8, CD008465.

Molassiotis, A., Fernandez-Ortega, P., Pud, D., Ozden, G., Scott, J.A., Panteli, V., Margulies, A., Browall, M., Magri, M., Selvekerova, S., Madsden, E., Milovics, L., Bruyns, I., Gudmundsdottir, G., Hummerston, S., Ahmad, A.M.A., Platin, N., Kearny, N., Patiraki, E. (2005). Use of complementary and alternative medicine in cancer patients: a European survey. Ann Oncol, 16, 655-663.

Molassiotis, A., Scott, J.A., Kearney, N., Pud, D., Magri, M., Selvekerova, S., Bruyns, I., Fernandez-Ortega, P., Panteli, V., Margulies, A., Gudmundsdottir, G., Milovics, L., Ozden, G., Platin, N., Patiraki, E. (2006). Complementary and alternative medicine usw in breast cancer patients in Europe. Support Care Cancer, 14(3), 260267.

Montazeri, A., Sajadian, A., Ebrahimi, M., Akbari, M.E. (2005). Depression and the use of complementary medicine among breast cancer patients. Support Care Cancer, 13, 339-342.

Münstedt, K., Hübner, J. (2013). Alternative Medizin bei Tumorerkrankungen. Onkologe, 19(2), 117-124.

Münstedt, K., Brüggemann, D., Jungi, W.F. (2007). Naturheilkunde und Komplementärmedizin in der Tumortherapie. Onkologe, 6(13), 528-533.

Musial, F., Büssing, A., Heusser, P., Choi, K.E., Ostermann, T. (2011). Mindfulness-based stress reduction for integrative cancer care: a summary of evidence. Forsch Komplementmed, 18(4), 192-202.

Myers, C.D., Walton, T., Small, B.J. (2008). The value of massage therapy in cancer care. Hematol Oncol Clin North Am, 22(4), 649-660.

Nagel, G., Hoyer, H., Katenkamp, D. (2004). Use of complementary and alternative medicine by patients with breast cancer: observations from a health care survey. Support Care Cancer, 12(11), 789-796.

Navo, M.A., Phan, I., Vaughan, C., Palmer, I.L., Michaud, L., Jones, K.I., Bodurka, D.C., Basen-Engquist, K., Hortobagyi, G.N., Kavanagh, J.I., Smith, J.A. (2004). An assessment of the utilization of complementary and alternative medication in women with gynecologic or breast malignancies. I Clin Oncol, 22, 671-677.

Nechuta, S., Lu, W., Chen, Z., Zheng, Y., Gu, K., Cai, H., Zheng, W., Shu, X.O. (2011). Vitamin supplement use during breast cancer treatment and survival: a prospective cohort study. Cancer Epidemiol Biomarkers Prev, 20(2), 262-271.

Neumann, M., Edelhäuser, F., Kreps, G.L., Scheffer, C., Lutz, G., Tauschel, D., Visser, A. (2010). Can patient-provider interaction increase the effectiveness of medical treatment or even substitute it? - an exploration on why and how to study the specific effect of the provider. Patient Educ Couns, 80(3), 307-314. 
0'Callaghan, V. (2011). Patients' perceptions of complementary and alternative medicine. Cancer Forum, 35(1), 44-47.

Oh, B., Butow, P., Mullan, B., Beale, P., Pavlakis, N., Rosenthal, D., Clarke, S. (2010a). The use and benefits resulting from the use of complementary and alternative medicine by cancer patients in Australia. Asia Pac ) Clin Oncol, 6(4), 342-349.

Oh, B., Butow, P., Mullan, B., Clarke, S., Tattersall, M., Boyer, M., Beale, P., Vardy, I., Pavlakis, N., Larke, L. (2010b). Patient-doctor communication: use of complementary and alternative medicine by adult patients with cancer. I Soc Integr Oncol, 8(2), 56-64.

Ostermann, T., Raak, C., Büssing, A. (2009). Survival of cancer patients treated with mistletoe extract (Iscador): a systematic literature review. BMC Cancer, 9:451.

Paltiel, 0., Avitzour, M., Peretz, T., Cherny, N., Kaduri, L., Pfeffer, R.M., Wagner, N., Soskolne, V. (2001). Determinants of the use of complementary therapies by patients with cancer. J Clin Oncol, 19, 2439-2448.

Paul, M., Davey, B., Senf, B., Stoll, C., Münstedt, K., Mücke, R., Micke, O., Prott, F..., Büntzel, I., Hübner, J. (2013). Patients with advanced cancer and their usage of complementary and alternative medicine. I Cancer Res Clin Oncol, 139(9):1515-22.

Pedersen, C.G., Christensen, S., Jensen, A.B., Zachariae, R. (2009). Prevalence, socio-demographic and clinical predictors of post-diagnostic utilisation of different types of complementary and alternative medicine (CAM) in a nationwide cohort of Danish women treated for primary breast cancer. Eur J Cancer, 45(18), 3172-3181.

Perabo, F.G.E., Löw, E.C., Siener, R., Ellinger, J., Müller, S.C., Bastian, P.J. (2009). Eine kritische Bewertung der Phytotherapie des Prostatakarzinoms. Urologe, 3, 270-283.

Peteet, J.R., Balboni, M.J. (2013). Spirituality and religion in oncology. CA Cancer I Clin, 63(4), 280-289.

Ponholzer, A., Struhal, G., Madersbacher, S. (2003). Frequent use of complementary medicine by prostate cancer patients. Eur Urol, 43(6), 604-608.

Porter, M., Kolva, E., Ahl, R., Diefenbach, M.A. (2008). Changing patterns of CAM use among prostate cancer patients two years after diagnosis: reasons for maintenanace or discontinuation. Complement Ther Med, 16(6), 318-324.

Rakovitch, E., Pignol, J.P., Chartier, C., Ezer, M., Verma, S., Dranitsaris, G., Clemons, M. (2005). Complementary and alternative medicine use is associated with an increased perception of breast cancer risk and death. Breast Cancer Res Treat, 90(2), 139-148.

Ramsey, S.D., Zeliadt, S.B., Blough, D.K., Fedorenko, C.R., Fairweather, M.E., McDermott, C.L., Penson, D.F., van den Eeden, S.K., Hamilton, A.S., Arora, N.K. (2012). Complementary and alternative medicine use, patientreported outcomes, and treatment satisfaction among men with localized prostate cancer. Urology, 79(5), 1034-1041.

Rausch, S.M., Winegardner, F., Kruk, K.M., Phatak, V., Wahner-Roedler, D.I., Bauer, B., Vincent, A. (2011). Complementary and alternative medicine: use and disclosure in radiation oncology community practice. Support Care Cancer, 19, 521-529.

Rhode, I., Fogoros, S., Zick, S., Wahl, H., Griffith, K.A., Huang, I., Liu, R. (2007). Ginger inhibits cell growth and modulates angiogenic factors in ovarian cancer cells. BMC Complement Altern Med, 7:44.

Richardson, M.A., Masse, L.C., Nanny, K., Sanders, C. (2004). Discrepant views of oncologists and cancer patients on complementary/alternative medicine. Support Care Cancer, 12(11), 797-804.

Richardson, I., Smith, J.E., McCall, G., Richardson, A., Pilkington, K., Kirsch, I. (2007). Hypnosis for nausea and vomiting in cancer chemotherapy: a systematic review of the research evidence. Eur / Cancer Care, 16, 402412.

Robert Koch Institut (RKI) (2010). Verbreitung von Krebserkrankungen in Deutschland. Entwicklung der Prävalenzen zwischen 1990 und 2010. Berlin (GBE Bund).

Roberts, C.S., Baker, F., Hann, D., Runfola, I., Witt, C., McDonald, J., Livingston, M.L., Ruiterman, I., Ampela, R., Kaw, O.C., Blanchard, C. (2005). Patient-physician communication regarding use of complementary therapies during cancer treatment. I Psychosoc Oncol, 23(4), 35-60.

Robotin, M., Holliday, C., Bensoussan, A. (2012). Defining research priorities in complementary medicine in oncology. Complement Ther Med, 20(5), 345-352.

Ross, L.E., Hall, I.J., Fairley, T.L., Taylor, Y.J., Howard, D.L. (2008). Prayer and self-reported health among cancer survivors in the United States, National Health Interview Survey, 2002. I Altern Complement Med, 14, 931938. 
Saquib, I., Madlensky, L., Kealey, S., Saquib, N., Natarajan, L., Newman, V.A., Patterson, R.E., Pierce, J.P. (2011). Classification of CAM use and its correlates in patients with early-stage breast cancer patients. Integr Cancer Ther, 10(2), 138-147.

Sarris, I., Byrne, G.J. (2011). A systematic review of insomnia and complementary medicine. Sleep Med Rev, 15(2), 99-106.

Saxe, G.A., Madlensky, L., Kealey, S., Wu, D.P., Freeman, K.L., Pierce, I.P. (2008). Disclosure to physicians of CAM use by breast cancer patients: findings from the Women's Healthy Eating and Living Study. Integr Cancer Ther, 7(3), 122-129.

Schernhammer, E.S., Haidinger, G., Waldhör, T., Vutuc, C. (2009). Attitudes about the use of complementary and alternative medicine in cancer treatment. I Altern Complement Med, 15(10), 1115-1120.

Schieman, C., Rudnik, L.R., Dixon, E., Sutherland, F., Bathe, O.F. (2009). Complementary and alternative medicine use among surgery, hepatobiliary surgery and surgical oncology patients. Can I Surg, 52(5), 422-426.

Schilling, G., Arnold, D. (2012). Therapiefolgen bei Krebs-Langzeitüberlebenden. Bundesgesundheitsbl, 55, 493500.

Schofield, P., Diggens, J., Charleson, C., Marigliani, R. (2010). Effectively discussing complementary and alternative medicine in a conventional oncology setting: Communication recommendations for clinicians. Pat Educ Counsel, 79(2), 143-151.

Schreiber, J.A., Brockopp, D.Y. (2012). Twenty-five years later - what do we know about religion/spirituality and psychological well-being among breast cancer survivors? A systematic review. J Cancer Surviv, 6(1), 82-94.

Seely, D.M., Weeks, L.C., Young, S. (2012). A systematic review of integrative oncology programs. Curr Oncol, 19, e436-e461.

Shelley, B.M., Sussman, A.L., Williams, R.L., Segal, A.R., Crabtree, B.F., Rios Net Clinicians (2009).'They don’t ask me so Idon't tell them': patient-clinician communication about traditional, complementary, and alternative medicine. Ann Fam Med, 7(2), 139-147.

Shneerson, C., Taskila, T., Gale, N., Greenfield, S., Chen, Y.F. (2013). The effect of complementary and alternative medicine on the quality of life of cancer survivors: A systematic review and meta-analyses. Complement Ther Med, 21(4), 417-429.

Shu, X.O., Zheng, Y., Cai, H., Gu, K., Chen, Z., Zheng, W., Lu, W. (2009). Soy food intake and breast cancer survival. JAMA, 302(22), 2437-2443.

Singh, H., Maskarinec, G., Shumay, D.M. (2005). Understanding the motivation for conventional and complementary/alternative medicine use among men with prostate cancer. Integr Cancer Ther, 4(2), 187-194.

Smith, K.B., Pukall, C.F. (2009). An evidence-based review of yoga as a complementary intervention for patients with cancer. Psychooncology, 18(5), 465-475.

Smithson, J., Paterson, C., Britten, N., Evans, M., Lewith, G. (2010a). Cancer patients' experiences of using complementary therapies: polarization and integration. J Health Serv Res Policy, 15(Suppl. 2), 54-61.

Smithson, I., Britten, N., Paterson, C., Lewith, G., Evans, M. (2010b). The experience of using complementary therapies after a diagnosis of cancer: A qualitative synthesis. Health, 20(10), 1-21.

Sood, A., Barton, D.L., Bauer, B.A., Loprinzi, C.L. (2007). A critical review of complementary therapies for cancerrelated fatigue. Integr Cancer Ther, 6(1), 8-13.

Strait, L., Furnham, A. (2012). The effect of modern health worries and psychological distress on complementary medicine use by breast cancer patients. / Clinic Res Bioeth, 3, 126.

Stratton, J., Godwin, M. (2011). The effect of supplemental vitamins and minerals on the development of prostate cancer: a systematic review and meta-analysis. Fam Pract, 28, 243-252.

Tascilar, M., de Jong, F.A., Verwej, I., Mathijssen, R.H.J. (2006). Complementary and alternative medicine during cancer treatment: Beyond innocence. Oncologist, 11, 732-741.

Tautz, E., Momm, F., Hasenburg, A., Güthlin, C. (2012). Use of complementary and alternative medicine in breast cancer patients and their experiences: A cross-sectional study. Eur / Cancer, 48(17), 3133-3139.

Trimborn, A., Senf, B., Münstedt, K., Büntzel, I., Micke, O., Prott, F.J., Wicker, S., Hübner, I. (2013). Attitude of employees of a university clinic to complementary and alternative medicine in oncology. Ann Oncol, 24(10), 2641-2645.

Tschuschke, V. (2008). Psychoonkologie. Naturheilkunde, 9, 823-840.

Tschuschke, V. (2013). Der Einfluss von Krankheitstheorien auf erwachsene Leukämie-Patienten. In: Hoefert, H.W., Brähler, E. (Hrsg.). Krankheitsvorstellungen von Patienten - Herausforderung für Medizin und Psychotherapie. Lengerich (Pabst), 139-142. 
van Patten, C.L., de Boer, J.G., Tomlinson Guns, E. (2008). Diet and dietary supplement intervention trials for the prevention of prostate cancer recurrence: A review of the randomized controlled trial evidence. I Urol, 180, 2314-2322.

Vehling, S., Koch, U., Ladehoff, N., Schön, G., Wegscheider, K., Heckl, U., Weis, J., Mehnert, A. (2012). Prävalenz affektiver und Angststörungen bei Krebs: Systematischer Literaturreview und Metaanalyse. Psychother Psych Med, 62(7), 249-258.

Verhoef, M.J., Boon, H.S., Page, S.A. (2008). Talking to cancer patients about complementary therapies: is it the physician's responsibility? Integr Oncol, 15(2), S88-S93.

Verhoef, M.J., Trojan, I., Armitage, G.D., Carlson, I., Hilsden, R.J. (2009). Complementary therapies for cancer patients: assessing information use and needs. Chron Dis Canada, 29(2), 80-88.

Wanchai, A., Armer, J.M., Stewart, B.R. (2010). Complementary and alternative medicine use among women with breast cancer: A systematic review. Clin I Oncol Nurs, 14(4), E45-E55.

Werneke, U., Ladenheim, D., McCarthy, T. (2004). Complementary alternative medicine for cancer: a review of effectiveness and safety. Cancer Ther, 2, 475-500.

Wilkinson, S.M., Love, S.B., Westcombe, A.M., Gambles, M.A., Burgess, C.C., Cargill, A., Young, T., Maher, E.J., Ramirez, A.J. (2007). Effectiveness of aromatherapy massage in the management of anxiety and depression in patients with cancer: A multicenter randomized controlled trial. I Clin Oncol, 25(5), 532-539.

Xu, L., Lao, L.X., Ge, A., Yu, S., Li, I., Mansky, P.J. (2007). Chinese herbal medicine for cancer pain. Integr Cancer Ther, 6(3), 208-234.

Yim, V.W., Ng, A.K., Tsang, H.W., Leung, A.Y. (2009). A review of the effects of aromatherapy for patients with depressive symptoms. I Altern Complement Med, 15(2), 187-195.

Yun, Y.H., Lee, M.K., Prark, S.M., Kim, Y.A., Lee, W.J., Lee, K.S., Choi, J.S., Jung, K.H., Do, Y.R., Kim, S.Y., Heo, D.S., Kim, H.T., Park, S.R. (2013). Effect of complementary and alternative medicine on the survival and healthrelated quality of life among terminally ill cancer patients: a prospective cohort study. Ann Oncol, 24(2), 489-494.

Zachariae, R., Johannessen, H. (2011). A methodological framework for evaluating the evidence for complementary and alternative medicine (CAM) for cancer. Cancer, 3, 773-788.

Zainal, N.Z., Booth, S., Huppert, F.A. (2013). The efficacy of mindfulness-based stress reduction on mental health of breast cancer patients: a meta-analysis. Psychooncology, 22(7), 1457-1465.

Zeliadt, S.B., Ramsey, S.D., Penson, D.F., Hall, I.J., Ekwueme, D.U., Stroud, L., Lee, I.W. (2006). Why do men choose one treatment over another? A review of patient decision making for localized prostate cancer. Cancer, 106(9), 1865-1874.

Zhang, Y., Peck, K., Spalding, M., Jones, B.G., Cook, R.L. (2012). Discrepancy between patients' use of and health providers' familiarity with CAM. Patient Educ Couns, 89(3), 399-404. 